\title{
Thermodynamic bounds on coherent transport in periodically driven conductors
}

\author{
Elina Potanina, ${ }^{1}$ Christian Flindt $\odot,{ }^{1}$ Michael Moskalets $\odot,{ }^{2}$ and Kay Brandner ${ }^{1,3,4,5}$ \\ ${ }^{1}$ Department of Applied Physics, Aalto University, 00076 Aalto, Finland \\ ${ }^{2}$ Department of Metal and Semiconductor Physics, NTU "Kharkiv Polytechnic Institute", \\ 61002 Kharkiv, Ukraine \\ ${ }^{3}$ Department of Physics, Keio University, 3-14-1 Hiyoshi, Kohoku-ku, Yokohama 223-8522, Japan \\ ${ }^{4}$ School of Physics and Astronomy, University of Nottingham, Nottingham NG7 2RD, United Kingdom \\ ${ }^{5}$ Centre for the Mathematics and Theoretical Physics of Quantum Non-equilibrium Systems, \\ University of Nottingham, Nottingham NG7 2RD, United Kingdom
}

(Received 23 September 2020; revised 26 January 2021; accepted 25 February 2021; published 14 April 2021)

\begin{abstract}
Periodically driven coherent conductors provide a universal platform for the development of quantum transport devices. Here, we lay down a comprehensive theory to describe the thermodynamics of these systems. We first focus on moderate thermoelectrical biases and low driving frequencies. For this linear response regime, we establish generalized Onsager-Casimir relations and an extended fluctuationdissipation theorem. Furthermore, we derive a family of thermodynamic bounds proving that any local matter or heat current puts a nontrivial lower limit on the overall dissipation rate of a coherent transport process. These bounds do not depend on system-specific parameters, are robust against dephasing, and involve only experimentally accessible quantities. They thus provide powerful tools to optimize the performance of mesoscopic devices and for thermodynamic inference, as we demonstrate by working out three specific applications. We then show that physically transparent extensions of our bounds hold also for strong biases and high frequencies. These generalized bounds imply a thermodynamic uncertainty relation that fully accounts for quantum effects and periodic driving. Moreover, they lead to a universal and operationally accessible bound on entropy production that can be readily used for thermodynamic inference and device engineering far from equilibrium. Connecting a broad variety of topics that range from thermodynamic geometry over thermodynamic uncertainty relations to quantum engineering, our work provides a unifying thermodynamic theory of coherent transport that can be tested and utilized with current technologies.
\end{abstract}

DOI: 10.1103/PhysRevX.11.021013

\section{INTRODUCTION}

Transport is a thermodynamic process, where gradients in intensive parameters such as chemical potential and temperature drive currents of extensive quantities like matter and energy. In macroscopic systems at high temperatures, this phenomenon can be understood as a result of frequent collisions between classical particles, which lead to random but biased changes of their direction of motion. This mechanism is know as diffusive transport [1]. Reducing the temperature of the system increases the mean free path that particles can travel between consecutive collisions. When this length scale becomes comparable to the dimensions of the conductor, as occurs in nanoscale

Published by the American Physical Society under the terms of the Creative Commons Attribution 4.0 International license. Further distribution of this work must maintain attribution to the author(s) and the published article's title, journal citation, and DOI.
Subject Areas: Condensed Matter Physics, Quantum Physics, Statistical Physics structures at millikelvin temperatures, coherent transport sets in [2]. This regime is governed by the laws of quantum mechanics and can no longer be described in terms of collisions between particles with well-defined positions and momenta.

Instead, coherent transport can be seen as arising from the unitary propagation of beams of carriers that are emitted and absorbed by distant thermal reservoirs and undergo elastic scattering within the conductor. This approach goes back to the pioneering work of Landauer [3] and has since evolved into a standard theoretical tool of mesoscopic physics [2,4]. In particular, it has been extended to systems that are subject to oscillating electromagnetic fields, where the scattering of beams is still coherent but no longer elastic, since carriers can exchange discrete amounts of energy with the driving fields [5-8].

On the experimental side, technological progress has made it possible to realize and control periodically driven coherent conductors with a high degree of precision. Today, these systems provide us with a versatile platform to test the 
basic principles of thermodynamics at small length and energy scales and to develop new mesoscopic devices such as parametric quantum pumps, which can be used to realize dynamical single-electron sources [9-19] as well as for metrological applications [20-22], and adiabatic quantum motors, which may provide motive power to future nanomachines [23-26].

These endeavors will require a powerful theoretical framework to describe the thermodynamics of coherent transport in the presence of both thermochemical biases and periodic driving. A suitable starting point for such a theory is provided by Onsager's irreversible thermodynamics [2729]. The key idea of this approach is to describe irreversible processes in terms of two types of variables: thermodynamic forces, which drive the process, and currents, which correspond to the system's response. This concept is universal in that it can be applied to macroscopic [27-29] and mesoscopic [30,31] systems alike. Moreover, it can be consistently expanded to include periodic driving. Specifically, in the context of coherent transport, this generalization can be achieved by introducing an additional force, which is proportional to the frequency of the applied fields, and an additional current, which corresponds to the flux of photons that is absorbed by the carriers inside the conductor [32].

Here, we show that this framework can further be underpinned by rigorous generalizations of two cornerstone results of classical irreversible thermodynamics: the Onsager-Casimir relations [27-29,33], which explain the interdependence between linear-response coefficients as a consequence of microscopic time-reversal symmetry, and the fluctuation-dissipation theorem, which connects these coefficients to equilibrium current fluctuations [34,35]. Focusing on moderate electric and thermal biases and slowly varying driving fields, we then set out to derive our first key result, the relations

$$
\sigma \geq \frac{N}{4 K^{x x}(N-1)}\left(J_{\alpha}^{x}\right)^{2},
$$

which bound the overall dissipation $\sigma$ caused by a coherent transport process in an $\mathrm{N}$-terminal conductor in terms of any period averaged matter current $J_{\alpha}^{\rho}$ or heat current $J_{\alpha}^{q}$. The coefficients $K^{\rho \rho}$ and $K^{q q}$ thereby depend only on the equilibrium temperature and chemical potential of the conductor. These bounds are stronger than the second law, which only requires $\sigma \geq 0$, and universal in that they do not involve any system-specific parameters.

In the second part of this article, we extend our theory to systems that are driven far away from equilibrium. This endeavor leads to our second key result, the relation

$\sigma \geq \sqrt{\left(P_{\alpha \alpha}^{\rho \rho}+2 T_{\alpha} / h\right)^{2}+2 \psi^{*}\left(J_{\alpha}^{\rho}\right)^{2}}-\left(P_{\alpha \alpha}^{\rho \rho}+2 T_{\alpha} / h\right)$,

which makes it possible to bound the total dissipation rate $\sigma$ by measuring the average $J_{\alpha}^{\rho}$ and the zero-frequency noise
$P_{\alpha \alpha}^{\rho \rho}$ of a single matter current along with the temperature $T_{\alpha}$ of the corresponding reservoir; $h$ denotes Planck's constant and $\psi^{*} \simeq 8 / 9$ is a numerical factor. Quite remarkably, the bound (2) holds for any coherent multiterminal conductor, arbitrary strong thermochemical biases, and arbitrary fast periodic driving fields.

Covering both thermal and mechanical driving, the relations (1) and (2) lead to nontrivial bounds on the figures of merit of cyclic nanomachines based on coherent conductors. In this respect, they advance an active line of research, which has so far mainly focused on steady-state devices and is driven by two major motivations [36-49]. First, universal bounds on figures such as efficiency or power consumption make it possible to quantitatively compare and optimize different theoretical models of mesoscopic devices. Second, such relations can be used in experiments to estimate quantities that cannot be measured directly, a strategy known as thermodynamic inference [50]. As we show by working out three specific applications, our theory provides powerful tools for both of these purposes. In particular, we provide a detailed analysis of parametric quantum pumps, for which we uncover a close connection between our approach and the concepts of thermodynamic geometry, a framework that recently proved very useful for optimizing slowly driven quantum thermal machines [51-55].

We proceed as follows. In Sec. II, we review the essentials of the scattering approach to coherent transport in periodically driven conductors and show how it can be furnished with a thermodynamic structure. We then work out the linear-response theory for this framework and lay down the corresponding generalizations of the OnsagerCasimir relations and the fluctuation-dissipation theorem in Sec. III. In Sec. IV, we derive the key relation (1) and discuss its range of validity. The purpose of Sec. V is to demonstrate the versatile applicability of our linearresponse results. To this end, we consider three different mesoscopic devices. As an introductory example, we analyze a simple model of a quantum generator, whereby we prove that our new bounds are tight. We then move on to parametric quantum pumps, for which we derive an explicit optimization principle by connecting our theory to the framework of thermodynamic geometry. Furthermore, we show how our bounds can be used for thermodynamic inference. Finally, we further illustrate this technique by applying it to adiabatic quantum motors. In the second major part of this article, Sec. VI, we show how the bounds (1) can be extended beyond the limits of the linear-response regime and derive our second key relation (2). We then put these results in context with recent developments on thermodynamic uncertainty relations. To this end, we work out a case study, which proves that our bounds on entropy production go significantly beyond earlier results. Finally, we discuss the implications of our theory for autonomous coherent conductors. We summarize our work in Sec. VII. 


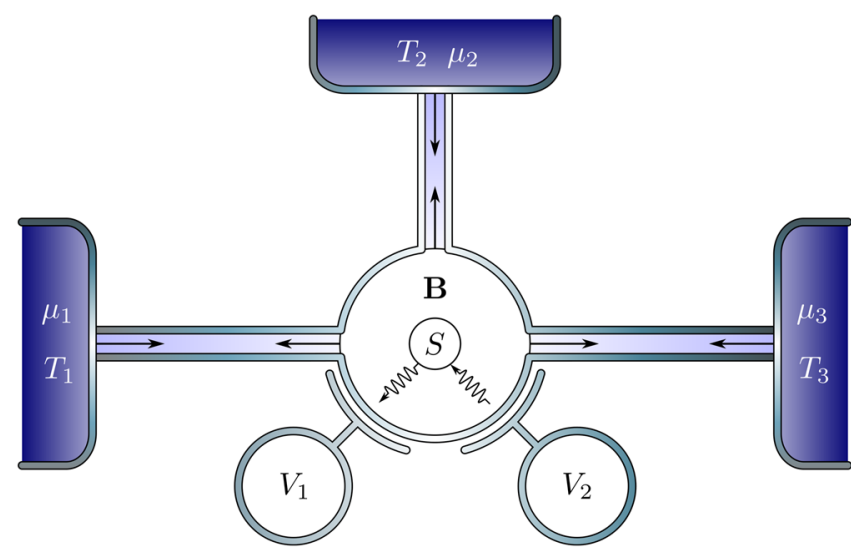

FIG. 1. Scattering approach to coherent transport. The figure shows a three-terminal conductor with two driving fields as a generic example for a multiterminal conductor. The system consists of a sample $S$, which is subject to a constant magnetic field $\mathbf{B}$, and three reservoirs with chemical potentials $\mu_{1}, \mu_{2}, \mu_{3}$ and temperatures $T_{1}, T_{2}, T_{3}$. Each reservoir injects a beam of carriers, which consists of a thermal mixture of plane waves propagating toward the sample. At the sample, each incoming beam is scattered into three outgoing beams. The phase relation between incoming and outgoing carriers is preserved, while their energy can change due to the exchange of photons with the periodic driving fields $V_{1}$ and $V_{2}$.

\section{SCATTERING APPROACH}

Scattering theory provides an elegant tool to describe coherent transport in periodically driven mesoscopic systems. In this approach, the conductor is divided into a sample region, where carriers may be subject to a periodically modulated potential and an external magnetic field, and a set of ideal leads. Each lead is connected to a reservoir, which injects a continuous beam of thermalized, noninteracting carriers. These beams propagate coherently through the system before being reabsorbed by the reservoirs; see Fig. 1. The emerging matter and energy currents are thus determined by the inelastic scattering amplitudes of the driven sample and the chemical potentials and temperatures of the reservoirs. In the following, we provide a brief review of this framework and its thermodynamic interpretation. Further details may be found in Refs. [5-8].

\section{A. Mean currents and fluctuations}

In the Heisenberg picture, the matter and energy currents that enter the system through the terminal $\alpha$ correspond to time-dependent operators $\hat{J}_{\alpha, t}^{\rho}$ and $\hat{J}_{\alpha, t}^{\varepsilon}$. The mean values and fluctuations of these currents are given by the general expressions

$$
J_{\alpha}^{u}=\lim _{t \rightarrow \infty} \frac{1}{t} \int_{0}^{t} d t^{\prime}\left\langle\hat{J}_{\alpha, t^{\prime}}^{u}\right\rangle
$$

$P_{\alpha \beta}^{u v}=\lim _{t \rightarrow \infty} \frac{1}{t} \int_{0}^{t} d t^{\prime} \int_{0}^{t} d t^{\prime \prime}\left\langle\left(\hat{J}_{\alpha, t^{\prime}}^{u}-J_{\alpha}^{u}\right)\left(\hat{J}_{\beta, t^{\prime \prime}}^{v}-J_{\beta}^{v}\right)\right\rangle$,

where $u, v=\rho, \varepsilon$ and angular brackets indicate the average over all quantum states of the injected carriers. Since the carriers are noninteracting, this average can be evaluated by treating the incoming beams as ideal fermionic quantum gases, which leads to the generalized Landauer-Büttiker formula,

$J_{\alpha}^{u}=\frac{1}{h} \int_{0}^{\infty} d E \sum_{\beta} \sum_{n}\left(\xi_{E}^{u} \delta_{\alpha \beta} \delta_{n 0}-\xi_{E n}^{u}\left|S_{E n, E}^{\alpha \beta}\right|^{2}\right) f_{E}^{\beta}$,

for the mean currents with $\xi_{E}^{\rho}=1$ and $\xi_{E}^{\varepsilon}=E$. Here, we have introduced the shorthand notation $E_{n} \equiv E+n \hbar \omega$, where $\hbar=h / 2 \pi$ denotes the reduced Planck constant and $\omega \equiv 2 \pi / \mathcal{T}$ the frequency of periodic driving fields acting on the sample. Thermodynamics enters Eq. (4) via the Fermi functions,

$$
f_{E}^{\alpha} \equiv \frac{1}{1+\exp \left[\left(E-\mu_{\alpha}\right) / T_{\alpha}\right]},
$$

where $\mu_{\alpha}$ and $T_{\alpha}$ are the chemical potential and temperature of the reservoir $\alpha$ and Boltzmann's constant is set to 1 throughout. The properties of the sample are encoded in the Floquet scattering amplitudes $S_{E n, E}^{\alpha \beta}$. These objects describe the transmission of an incoming carrier with energy $E$ from the terminal $\beta$ to the terminal $\alpha$ under the absorption of $n$ photons with energy $\hbar \omega$. Note that, for simplicity, we assume that each lead supports only one transport channel. Furthermore, we use the convention that the photoncounting index runs over all integers and that the Floquet scattering amplitudes are zero if one of their energy arguments is negative.

The current fluctuations (3b) can be evaluated in the same way as the mean currents. The resulting formula involves two contributions, $P_{\alpha \beta}^{u v}=D_{\alpha \beta}^{u v}+R_{\alpha \beta}^{u v}$, which are given by

$$
\begin{aligned}
& D_{\alpha \beta}^{u v}=\frac{1}{h} \int_{0}^{\infty} d E \sum_{n}\left(A_{n, E}^{u v, \alpha \beta}+A_{n, E}^{v u, \beta \alpha}+\delta_{\alpha \beta} B_{n, E}^{u v, \alpha}\right), \\
& R_{\alpha \beta}^{u v}=\frac{1}{2 h} \int_{0}^{\infty} d E \sum_{\gamma \delta} \sum_{n} C_{n, E}^{u \alpha, \gamma \delta} C_{n, E}^{v \beta, \gamma \delta *}
\end{aligned}
$$

with

$$
\begin{aligned}
A_{n, E}^{u v, \alpha \beta} & \equiv \xi_{E}^{u} \xi_{E}^{v} \delta_{\alpha \beta} \delta_{n 0} f_{E}^{\prime \alpha}-\xi_{E n}^{u} \xi_{E}^{v}\left|S_{E n, E}^{\alpha \beta}\right|^{2} f_{E}^{\prime \beta} \\
B_{n, E}^{u v, \alpha} & \equiv \sum_{\gamma} \xi_{E n}^{u} \xi_{E n}^{v}\left|S_{E n, E}^{\alpha \gamma}\right|^{2}\left(f_{E}^{\prime \gamma}-f_{E n}^{\prime \alpha}\right) \\
C_{n, E}^{u \alpha, \beta \gamma} & \equiv \sum_{m} \xi_{E m}^{u} S_{E m, E n}^{\alpha \gamma} S_{E m, E}^{\alpha \beta *}\left(f_{E}^{\beta}-f_{E n}^{\gamma}\right),
\end{aligned}
$$


and $f_{E}^{\prime \alpha} \equiv f_{E}^{\alpha}\left(1-f_{E}^{\alpha}\right)$. The thermal, or Nyquist-Johnson, noise $D_{\alpha \beta}^{u v}$ thereby arises from thermal fluctuations in the injected beams of carriers and vanishes in the zero-temperature limit. By contrast, the shot noise $R_{\alpha \beta}^{u v}$ stems from the probabilistic nature of carrier transmissions through the sample, and therefore persists at zero temperature.

\section{B. Unitarity and time-reversal symmetry}

The Floquet scattering amplitudes generally depend on the structure of the sample and the applied driving protocols $\mathbf{V}_{t}$, where $\mathbf{V} \equiv\left\{V_{j}\right\}$ denotes the set of external control parameters. Still, they obey two universal relations, which follow from fundamental principles. First, the unitarity conditions,

$$
\begin{aligned}
& \sum_{\alpha} \sum_{n} S_{E n, E}^{\alpha \beta} S_{E n, E m}^{\alpha \gamma *}=\delta_{\beta \gamma} \delta_{m 0}, \\
& \sum_{\alpha} \sum_{n} S_{E, E n}^{\beta \alpha} S_{E m, E n}^{\gamma \alpha *}=\delta_{\beta \gamma} \delta_{m 0},
\end{aligned}
$$

ensure the conservation of probabilities in individual scattering events. Second, the invariance of Schrödinger's equation under time reversal implies the symmetry

$$
S_{E n, E}^{\alpha \beta}=\mathrm{T}_{\mathbf{B}} \mathbf{T}_{\mathbf{V}} S_{E, E n}^{\beta \alpha},
$$

where the symbolic operators $T_{\mathbf{B}}$ and $T_{\mathbf{V}}$ indicate the reversal of external magnetic fields and driving protocols, respectively. Note that, while the unitarity conditions (8) apply to the scattering amplitudes of any given sample, the symmetry relation (9) connects the scattering amplitudes of two different systems that are related to each other by time reversal.

\section{Thermodynamics}

The thermodynamics of coherent transport can be developed from the conservation laws,

$$
\sum_{\alpha} J_{\alpha}^{\rho}=0 \quad \text { and } \quad \Pi_{\mathrm{ac}}+\sum_{\alpha} J_{\alpha}^{\varepsilon}=0
$$

which can be easily verified using generalized LandauerBüttiker formula (4) and the unitarity conditions (8). They reflect the fact that neither matter nor energy is accumulated in the sample over a full cycle. The quantity

$$
\Pi_{\mathrm{ac}} \equiv-\sum_{\alpha} J_{\alpha}^{\varepsilon}=\frac{1}{\mathcal{T}} \int_{0}^{\infty} d E \sum_{\alpha \beta} \sum_{n} n\left|S_{E n, E}^{\alpha \beta}\right|^{2} f_{E}^{\beta}
$$

thereby corresponds to the average mechanical power that is absorbed by the carriers from the driving fields.

Combining the conservation laws (10), leads to the first law of thermodynamics:

$$
\Pi_{\mathrm{ac}}+\Pi_{\mathrm{el}}=-\sum_{\alpha} J_{\alpha}^{q}
$$

Here, the electrical power $\Pi_{\mathrm{el}}$, which is generated by matter currents flowing in the direction of chemical potential gradients, and the heat currents entering the conductor from the reservoirs, $J_{\alpha}^{q}$, are given by

$$
\Pi_{\mathrm{el}} \equiv \sum_{\alpha} \mu_{\alpha} J_{\alpha}^{\rho} \quad \text { and } \quad J_{\alpha}^{q} \equiv J_{\alpha}^{\varepsilon}-\mu_{\alpha} J_{\alpha}^{\rho}
$$

Since the transfer of carriers through the system is coherent, and thus reversible, dissipation occurs only in the reservoirs due to the influx and outflux of heat. Hence, the total rate of entropy production is given by

$$
\begin{aligned}
\sigma & \equiv-\sum_{\alpha} J_{\alpha}^{q} / T_{\alpha} \\
& =\frac{1}{h} \int_{0}^{\infty} d E \sum_{\alpha \beta} \sum_{n}\left(\frac{E n-\mu_{\alpha}}{T_{\alpha}}-\frac{E-\mu_{\beta}}{T_{\beta}}\right)\left|S_{E n, E}^{\alpha \beta}\right|^{2} f_{E}^{\beta} .
\end{aligned}
$$

This expression is non-negative for any temperature and chemical potential profiles and any set of Floquet scattering amplitudes [8]. The scattering formalism is therefore consistent with the second law of thermodynamics, which requires $\sigma \geq 0$.

\section{LINEAR RESPONSE}

In irreversible thermodynamics, transport is described in terms of thermodynamic forces, or affinities, which correspond to gradients of intensive variables, such as temperature, and currents of extensive quantities like energy [29]. Every affinity forms a conjugate pair with a specific current such that the products of these pairs add up to the total rate of entropy production in the system. Close to equilibrium, the currents become linear functions of the affinities with the corresponding response coefficients obeying two universal relations: the Onsager-Casimir symmetry, which connects reciprocal coefficients $[27,28,33]$, and the fluctuation-dissipation theorem, which relates them to equilibrium current fluctuations [34,35]. These results are well established for stationary coherent transport in mesoscopic systems $[30,31,56]$. In the following, we show how they can be extended to systems with periodic driving by further developing the approach that was proposed in Ref. [32].

\section{A. Affinities}

The affinities for the matter and heat currents are given by the thermochemical gradients,

$$
F_{\alpha}^{\rho} \equiv\left(\mu_{\alpha}-\mu\right) / T \quad \text { and } \quad F_{\alpha}^{q} \equiv 1 / T-1 / T_{\alpha},
$$

where $\mu$ and $T$ are the reference chemical potential and temperature. Using these definitions and the conservation 
laws (10), the total rate of entropy production (14) can be written as

$$
\sigma=\Pi_{\mathrm{ac}} / T+\sum_{\alpha} \sum_{x} F_{\alpha}^{x} J_{\alpha}^{x}
$$

with $x=\rho, q$. Upon recalling the expression (11) for the average mechanical power, this result suggests that we introduce the photon current,

$$
J^{\omega} \equiv \Pi_{\mathrm{ac}} / \hbar \omega=\frac{1}{h} \int_{0}^{\infty} d E \sum_{\alpha \beta} \sum_{n} n\left|S_{E n, E}^{\alpha \beta}\right|^{2} f_{E}^{\beta},
$$

and the corresponding affinity $F^{\omega} \equiv \hbar \omega / T$ such that $\sigma$ assumes the canonical bilinear form:

$$
\sigma=J^{\omega} F^{\omega}+\sum_{\alpha} \sum_{x} J_{\alpha}^{x} F_{\alpha}^{x}=\sum_{A} J_{A} F_{A} .
$$

Here, we use capital roman letters to denote compound indices covering both thermochemical and mechanical quantities, i.e., $A=\{(x, \alpha)\}, \omega$.

Two remarks are in order. First, the interpretation of $J^{\omega}$ as flux of photons derives from the fact that the carriers and the driving fields exchange only discrete amounts of energy. This phenomenon, which, on the technical level, is a consequence of the Floquet theorem [8], is a manifestation of the laws of quantum mechanics and has no counterpart in classical mechanics [57]. Second, to achieve a thermodynamic unification of steady-state and periodic driving, the driving frequency is treated as a thermodynamic force in Eq. (18). This approach, which was proposed in Ref. [32], is more suitable for coherent transport than using the amplitude of the time-dependent fields as an effective affinity, a scheme that has proved very useful for systems obeying stochastic dynamics [58-62]. In particular, as we show next, the frequency-based approach enables a nontrivial linear-response theory, while a perturbation theory in the driving strength leads only to a trivial decoupling of thermochemical currents and mechanical driving; see the Appendix A 2 for details.

\section{B. Kinetic coefficients}

The kinetic coefficients that govern the relation between currents and affinities in the linear-response regime are defined as

$$
L_{A B}=\left.\partial_{F_{B}} J_{A}\right|_{\mathrm{eq}},
$$

where the notation $\left.\cdots\right|_{\text {eq }}$ indicates the limit $F_{A} \rightarrow 0$. To calculate the coefficients (19), we first observe that the Fermi functions of the reservoirs are given by

$$
f_{E}^{\alpha} \simeq f_{E}+\left(F_{\alpha}^{\rho}+(E-\mu) F_{\alpha}^{q}\right) f_{E}^{\prime},
$$

with $\left.f_{E} \equiv f_{E}^{\alpha}\right|_{\mathrm{eq}}$ and $\left.f_{E}^{\prime} \equiv f_{E}^{\prime \alpha}\right|_{\mathrm{eq}}=f_{E}\left(1-f_{E}\right)$, up to second-order corrections in the thermochemical affinities. Second, we recall that the Floquet scattering amplitudes admit the low-frequency expansion $[7,63]$ :

$$
\begin{aligned}
S_{E n, E m}^{\alpha \beta} \simeq & \frac{1}{\mathcal{T}} \int_{0}^{\mathcal{T}} d t\left(\mathcal{S}_{E, \mathbf{V} t}^{\alpha \beta}+\hbar \omega \frac{n+m}{2} \partial_{E} \mathcal{S}_{E, \mathbf{V} t}^{\alpha \beta}\right. \\
& \left.+\hbar \omega \mathcal{A}_{E, t}^{\alpha \beta}\right) e^{i(n-m) \omega t} .
\end{aligned}
$$

Here, the frozen scattering amplitudes $\mathcal{S}_{E, \mathbf{V}}^{\alpha \beta}$ describe the transmission of carriers with energy $E$ at fixed parameters V. The correction term $\mathcal{A}_{E, t}^{\alpha \beta}$ is required to ensure that the right-hand side of Eq. (21) obeys the unitarity condition (8). In general, the approximation (21) is applicable if the driving fields vary only slightly during the average dwell time $\tau_{\mathrm{dw}}$ of carriers inside the sample. This timescale is connected to the typical energy range $\delta_{E}$ over which the frozen scattering amplitudes change by the relation $\tau_{\mathrm{dw}}=\hbar / \delta_{E}$; for details, see Refs. [7,64,65].

Using Eqs. (20) and (21), the kinetic coefficients (19) can be determined as

$$
\begin{aligned}
& L_{\alpha \beta}^{x y}=\frac{1}{h} \int_{0}^{\infty} d E \zeta_{E}^{x} \zeta_{E}^{y}\left(\delta_{\alpha \beta}-\left\langle\left\langle\left|\mathcal{S}_{E, \mathbf{V}}^{\alpha \beta}\right|^{2}\right\rangle\right\rangle\right) f_{E}^{\prime}, \\
& L_{\alpha}^{x \omega}=\frac{1}{h} \int_{0}^{\infty} d E \zeta_{E}^{x} \zeta^{\omega} \sum_{\beta} \operatorname{Im}\left[\left\langle\left\langle\dot{\mathcal{S}}_{E, \mathbf{V}}^{\alpha \beta} \mathcal{S}_{E, \mathbf{V}}^{\alpha \beta *}\right\rangle\right] f_{E}^{\prime},\right. \\
& L_{\alpha}^{\omega x}=\frac{1}{h} \int_{0}^{\infty} d E \zeta_{E}^{x} \zeta^{\omega} \sum_{\beta} \operatorname{Im}\left[\left\langle\left\langle\mathcal{S}_{E, \mathbf{V}}^{\beta \alpha} \dot{\mathcal{S}}_{E, \mathbf{V}}^{\beta \alpha *}\right\rangle\right] f_{E}^{\prime},\right. \\
& L^{\omega \omega}=\frac{1}{2 h} \int_{0}^{\infty} d E\left(\zeta^{\omega}\right)^{2} \sum_{\alpha \beta}\left\langle\left\langle\left.\dot{\mathcal{S}}_{E, \mathbf{V}}^{\alpha \beta}\right|^{2}\right\rangle\right\rangle f_{E}^{\prime},
\end{aligned}
$$

with $\zeta_{E}^{\rho} \equiv 1, \zeta_{E}^{q} \equiv E-\mu, \zeta^{\omega} \equiv 1 / \omega$, dots indicating time derivatives, and double brackets denoting the time average over one period, $\langle\langle\cdots\rangle\rangle \equiv 1 / \mathcal{T} \int_{0}^{\mathcal{T}} d t \ldots$; for details, see Appendix A 1. Notably, the expressions (22) do not depend on the corrections $\mathcal{A}_{E, t}^{\alpha \beta}$ as a result of the unitarity condition (8). Instead, they involve only the frozen scattering amplitudes $\mathcal{S}_{E, \mathbf{V}}^{\alpha \beta}$, which are generally much easier to obtain than the full Floquet scattering amplitudes.

The linear response regime with respect to the affinities $F_{\alpha}^{\rho}, F_{\alpha}^{q}$, and $F^{\omega}$ is defined by the three conditions,

$F_{\alpha}^{\rho} \ll \mu / T, \quad F_{\alpha}^{q} \ll 1 / T, \quad$ and $\quad F^{\omega} \ll \delta_{E} / T$,

under which the currents obey the kinetic equations:

$$
J_{A}=\sum_{B} L_{A B} F_{B}
$$


This result extends the conventional framework of linearirreversible thermodynamics to periodically driven coherent conductors. We note that, at low temperatures, the function $f_{E}^{\prime}$ in Eqs. (22) is sharply peaked around $\mu$. The transmission of carriers then occurs only at energies close to the Fermi edge. It is therefore typically sufficient to require that the slow-driving condition $F^{\omega} \ll \delta_{E} / \hbar$ is obeyed at $E \simeq \mu$ for the kinetic equations (24) to be valid.

\section{Onsager-Casimir relations}

The Onsager-Casimir, or reciprocal, relations between linear-response coefficients follow from the symmetry of microscopic dynamics under time reversal. This principle enters stationary scattering theory through the property

$$
\mathcal{S}_{E, \mathbf{V}}^{\alpha \beta}=\mathrm{T}_{\mathbf{B}} \mathcal{S}_{E, \mathbf{V}}^{\beta \alpha}
$$

of the frozen scattering amplitudes [8], which, together with the formulas (22), implies the relations

$$
\mathrm{T}_{\mathbf{B}} L_{\alpha \beta}^{x y}=L_{\beta \alpha}^{y x} \quad \text { and } \quad \mathrm{T}_{\mathbf{B}} L_{\alpha}^{\omega x}=-L_{\alpha}^{x \omega} ;
$$

recall Sec. II B for the definition of $T_{\mathbf{B}}$ and $T_{\mathbf{V}}$. Hence, while the thermochemical coefficients obey the conventional Onsager-Casimir symmetry, the cross-coefficients that couple either to the mechanical affinity or the photon current are antisymmetric. The original symmetry can, however, be restored for all kinetic coefficients by reversing both magnetic fields and driving protocols. That is, we have the generalized Onsager-Casimir relations [66]

$$
\mathrm{T}_{\mathbf{B}} \mathrm{T}_{\mathbf{V}} L_{A B}=L_{B A} .
$$

Notably, this result implies that, if the driving protocols are symmetric, i.e., if $\mathbf{V}_{t}=\mathbf{V}_{-t}$, the mechanical and thermochemical currents and affinities decouple, since $L_{\alpha}^{\chi \omega}=L_{\alpha}^{\omega x}=0$.

\section{Fluctuation-dissipation theorem}

The fluctuation-dissipation theorem provides a link between kinetic coefficients and equilibrium current fluctuations. For periodically driven coherent conductors, this connection can be established as follows. First, we note that, with respect to the fluctuations of matter and energy currents $P_{\alpha \beta}^{u v}$, which are spelled out in Eqs. (6) and (7), the joint fluctuations of matter and heat currents are given by

$$
P_{\alpha \beta}^{x y}=\sum_{u v} c_{\alpha}^{x u} c_{\beta}^{y v} P_{\alpha \beta}^{u v},
$$

with $c_{\alpha}^{\rho u} \equiv \delta_{u \rho}$ and $c_{\alpha}^{q u} \equiv \delta_{u \varepsilon}-\mu_{\alpha} \delta_{u \rho}$ [8]. Next, we observe that the shot noise Eq. (6b) vanishes in equilibrium, $\left.R_{\alpha \beta}^{u v}\right|_{\text {eq }}=0$. Therefore, we have

$$
\left.P_{\alpha \beta}^{x y}\right|_{\mathrm{eq}}=\left.\sum_{u v}\left(c_{\alpha}^{x u} c_{\beta}^{y v} D_{\alpha \beta}^{u v}\right)\right|_{\mathrm{eq}}=L_{\alpha \beta}^{x y}+L_{\beta \alpha}^{y x},
$$

as can be easily verified by inspection. For systems without magnetic fields, we thus recover the standard result $P_{\alpha \beta}^{x y}=$ $2 L_{\alpha \beta}^{x y}$ by using the symmetry (26).

To derive a fluctuation-dissipation relation for the coefficients $L_{\alpha}^{\omega x}, L_{\alpha}^{x \omega}$, and $L^{\omega \omega}$, we have to consider the fluctuations involving the photon current, which, due to energy conservation, can be obtained from the sum rules:

$$
P_{\alpha}^{u \omega}=-\sum_{\beta} P_{\alpha \beta}^{u \varepsilon} / \hbar \omega \text { and } P^{\omega \omega}=\sum_{\alpha \beta} P_{\alpha \beta}^{\varepsilon \varepsilon} /(\hbar \omega)^{2} .
$$

Inserting the expressions (6) and (7) for the thermal and the shot noise and using the unitarity conditions (8) for the Floquet scattering amplitudes yields the explicit results $P_{\alpha}^{u \omega}=D_{\alpha}^{u \omega}+R_{\alpha}^{u \omega}$ and $P^{\omega \omega}=D^{\omega \omega}+R^{\omega \omega}$, with

$D_{\alpha}^{u \omega}=\frac{1}{h} \int_{0}^{\infty} d E \sum_{\beta} \sum_{n}\left(\xi_{E}^{u} A_{n, E}^{\omega 1, \beta \alpha}-\xi_{E n}^{u} A_{n, E}^{\omega 1, \alpha \beta}\right)$,

$D^{\omega \omega}=\frac{1}{h} \int_{0}^{\infty} d E \sum_{\alpha \beta} \sum_{n} A_{n, E}^{\omega 2, \alpha \beta}$,

$R_{\alpha}^{u \omega}=\frac{1}{2 h} \int_{0}^{\infty} d E \sum_{\gamma \delta} \sum_{n} C_{n, E}^{u \alpha, \gamma \delta} C_{n, E}^{\omega, \gamma \delta *}$

$R^{\omega \omega}=\frac{1}{2 h} \int_{0}^{\infty} d E \sum_{\gamma \delta} \sum_{n} C_{n, E}^{\omega, \gamma \delta} C_{n, E}^{\omega, \gamma \delta *}$

and

$$
\begin{aligned}
A_{n, E}^{\omega k, \alpha \beta} & \equiv n^{k}\left|S_{E n, E}^{\alpha \beta}\right|^{2} f_{E}^{\prime \beta}, \\
C_{n, E}^{\omega, \beta \gamma} & \equiv \sum_{\alpha} \sum_{m} m S_{E m, E n}^{\alpha \gamma} S_{E m, E}^{\alpha \beta *}\left(f_{E}^{\beta}-f_{E n}^{\gamma}\right) .
\end{aligned}
$$

The expression (32b) shows that $\left.R_{\alpha}^{u \omega}\right|_{\mathrm{eq}}=0$. After switching from energy to heat currents, we are therefore left with [67]

$$
\begin{aligned}
& \left.P_{\alpha}^{x \omega}\right|_{\mathrm{eq}}=\left.\sum_{u}\left(c_{\alpha}^{x u} D_{\alpha}^{u \omega}\right)\right|_{\mathrm{eq}}=L_{\alpha}^{x \omega}+L_{\alpha}^{\omega x}, \\
& \left.P^{\omega \omega}\right|_{\mathrm{eq}}=\left.D^{\omega \omega}\right|_{\mathrm{eq}}=2 L^{\omega \omega} .
\end{aligned}
$$

Hence, quite remarkably, the mechanical kinetic coefficients and the equilibrium fluctuations of the photon current obey the same relations as their thermochemical counterparts. This result is summarized by the extended fluctuation-dissipation theorem,

$$
\left.P_{A B}\right|_{\mathrm{eq}}=L_{A B}+L_{B A},
$$


which completes our linear-response framework. Notably, it implies, together with the symmetries (26) and (27), that $\left.P_{\alpha}^{x \omega}\right|_{\text {eq }}=0$ for systems without a magnetic field or with symmetric driving protocols. That is, equilibrium correlations between the photon current and the thermochemical currents are ultimately a result of broken time-reversal symmetry.

\section{NEW BOUNDS}

According to the second law, the rate of entropy production $\sigma$, which provides a measure for the thermodynamic cost of irreversible transport, cannot be negative. However, the laws of thermodynamics do not determine how much entropy must be generated to sustain a given current, as the following argument shows. In linear response, the currents $J_{A}$ can be divided into an irreversible and a reversible contributions given by [38]

$J_{A}^{\mathrm{irr}} \equiv \sum_{B} \frac{L_{A B}+L_{B A}}{2} F_{B}, \quad J_{A}^{\mathrm{rev}} \equiv \sum_{B} \frac{L_{A B}-L_{B A}}{2} F_{B}$.

Using these variables, the rate of entropy production (18) can be expressed as

$$
\sigma=\sum_{A} J_{A}^{\mathrm{irr}} F_{A} .
$$

Hence, the reversible currents, which, due to the generalized Onsager-Casimir relation (27), exist only in systems with broken time-reversal symmetry, do not contribute to $\sigma$. As a result, transport without dissipation seems to be possible in situations where $J_{A}^{\text {irr }}=0$ and at least one reversible current is finite $[37,68]$. This a priori surprising observation prompts the question of whether their might be stronger bounds on thermal currents than the second law. In the following, we first derive such bounds for coherent transport and then prove their robustness against dephasing.

\section{A. Coherent transport}

Our new bounds follow from the unitarity conditions for the frozen scattering amplitudes [7],

$$
\sum_{\alpha} \mathcal{S}_{E, \mathbf{V}}^{\alpha \beta} \mathcal{S}_{E, \mathbf{V}}^{\alpha \gamma *}=\sum_{\alpha} \mathcal{S}_{E, \mathbf{V}}^{\beta \alpha} \mathcal{S}_{E, \mathbf{V}}^{\gamma \alpha *}=\delta_{\beta \gamma},
$$

which ensure probability conservation, and the sum rule

$$
\sum_{\alpha \beta}\left\langle\left\langle\dot{\mathcal{S}}_{E, \mathbf{V}}^{\alpha \beta} \mathcal{S}_{E, \mathbf{V}}^{\alpha \beta *}\right\rangle=0\right.
$$

which plays the role of a gauge condition fixing the global phase of the frozen scattering amplitudes; see Lemma 3 of Appendix B. Together, they lead to the sum rules $\sum_{\alpha} L_{\alpha \beta}^{x y}=0$ and $\sum_{\alpha} L_{\alpha}^{x \omega}=0$ for the kinetic coefficients (22), which imply the conservation laws:

$$
\sum_{\alpha} J_{\alpha}^{\rho}=0 \quad \text { and } \quad \sum_{\alpha} J_{\alpha}^{q}=0
$$

Note that the conservation law for the heat currents is consistent with the first law (12) in linear response, since the electrical and the mechanical power, $\Pi_{\mathrm{el}}$ and $\Pi_{\mathrm{ac}}$, are of second order in the affinities.

As a technical tool, we now define the quadratic form,

$$
\Xi \equiv \sigma+\sum_{\alpha} \sum_{x} J_{\alpha}^{x} G_{\alpha}^{x}+\sum_{\alpha} \sum_{x y} K^{x y} G_{\alpha}^{x} G_{\alpha}^{y},
$$

where the $G_{\alpha}^{x}$ are real but otherwise arbitrary variables and the coefficients

$$
K^{x y} \equiv \frac{1}{2 h} \int_{0}^{\infty} d E \zeta_{E}^{x} \zeta_{E}^{y} f_{E}^{\prime}
$$

have been chosen such that $\Xi$ is positive semidefinite. To verify this property, we expand the rate of entropy production $\sigma$ and the thermochemical currents $J_{\alpha}^{x}$ in the affinities using Eq. (18) and the kinetic equations (24). Upon inserting the expressions (22) for the kinetic coefficients, and applying the unitarity conditions (37), Eq. (40) can thus be rewritten in the form

$$
\Xi=\frac{1}{2 h} \int_{0}^{\infty} d E \sum_{\alpha \beta}\left\langle\left\langle\left|X_{E}^{\alpha \beta} \mathcal{S}_{E, \mathbf{V}}^{\alpha \beta}-i X^{\omega} \dot{\mathcal{S}}_{E, \mathbf{V}}^{\alpha \beta}\right|^{2}\right\rangle\right\rangle_{E}^{\prime},
$$

with $X_{E}^{\alpha \beta} \equiv \sum_{x} \zeta_{E}^{x}\left(F_{\alpha}^{x}-F_{\beta}^{x}+G_{\alpha}^{x}\right)$ and $X^{\omega} \equiv \zeta^{w} F^{\omega}$, which proves that $\Xi$ cannot be negative, since $f_{E}^{\prime} \geq 0$.

This result leads to a whole family of bounds on $\sigma$, which can be extracted as follows. We first set $G_{\alpha}^{q}=0$ and minimize the right-hand side of Eq. (40) with respect to the variables $G_{\alpha}^{\rho}$. After repeating this step with the roles of $G_{\alpha}^{q}$ and $G_{\alpha}^{\rho}$ interchanged, we arrive at the cumulative bound:

$$
\sigma \geq \frac{1}{4 K^{x x}} \sum_{\alpha}\left(J_{\alpha}^{x}\right)^{2}
$$

To obtain bounds that involve only a single current, we rewrite Eq. (43) as

$$
\sigma \geq \frac{\left(J_{\alpha}^{x}\right)^{2}}{4 K^{x x}}+M_{\alpha}^{x}, \quad \text { with } \quad M_{\alpha}^{x} \equiv \frac{1}{4 K^{x x}} \sum_{\beta \neq \alpha}\left(J_{\beta}^{x}\right)^{2} .
$$

Minimizing $M_{\alpha}^{x}$ with respect to the currents $J_{\beta}^{x}$ while taking into account the conservation laws (39) as a constraint yields $M_{\alpha}^{x} \geq\left(J_{\alpha}^{x}\right)^{2} / 4 K^{x x}(N-1)$ and, thus,

$$
\sigma \geq \frac{N}{4 K^{x x}(N-1)}\left(J_{\alpha}^{x}\right)^{2},
$$


where $N$ is the number of terminals of the conductor and the inequality holds for any pair of indices $x$ and $\alpha$.

This relation constitutes a key result of this paper. Going beyond the second law, it shows that, regardless of the behavior of the system under time reversal, any matter or heat current comes at the price of a minimal rate of entropy production that is proportional to the square of this current. The positive coefficients $K^{x x}$ thereby depend only on the equilibrium properties of the reservoirs. Specifically, they are given by

$$
\begin{aligned}
K^{\rho \rho}= & \frac{T \varphi}{2 h(1+\varphi)} \leq \frac{T}{2 h}, \\
K^{q q}= & \frac{\pi^{2} T^{3}}{6 h}-\frac{T^{3}(\ln [\varphi])^{2}}{2 h(1+\varphi)}-\frac{T^{3} \ln [\varphi] \ln [1+1 / \varphi]}{h} \\
& +\frac{T^{3} \operatorname{Li}_{2}[-1 / \varphi]}{h} \leq \frac{\pi^{2} T^{3}}{6 h},
\end{aligned}
$$

where $\mathrm{Li}_{2}$ denotes the dilogarithm, $\varphi \equiv \exp [\mu / T]$ the equilibrium fugacity of the reservoirs, and the inequalities are saturated in the limit $\varphi \rightarrow \infty$, which is practically realized in mesoscopic conductors.

\section{B. Dephasing}

Coherent transport is characterized by a fixed phase relation between incoming and outgoing carriers. Under realistic conditions, however, phase-breaking mechanisms such as carrier-carrier or carrier-phonon interactions can hardly be completely suppressed. Probe terminals provide an elegant way to account for such effects [69]. In this approach, virtual reservoirs are attached to the conductor, whose temperature and chemical potential are adjusted such that they do not exchange matter or heat with the remaining system on average, but rather act as a source of dephasing.

On the technical level, the virtual reservoirs differ from physical ones only in that their affinities are fixed by the conditions of zero mean currents. The bounds (43), however, were derived without any assumptions on the affinities. They therefore apply also to systems with arbitrary many probe terminals, where only the currents between physical reservoirs contribute to the sum on the right; those currents flowing into the virtual reservoirs are zero by construction. Since the conservation laws (39) are likewise not affected by the probe terminals, also the bounds (45) remain valid with $N$ referring to the number of real terminals. Hence, our new bounds are robust against dephasing and hold even in the limit of fully incoherent transmission [69].

\section{APPLICATIONS}

In this section, we discuss three different quantum devices to explore the practical implications of our new bounds on coherent transport and their potential as tools of thermodynamic inference. As a first example, we consider a basic model of a magnetic-flux driven quantum generator, which was proposed in Ref. [36]. This case study serves as a simple illustration of our general theory and shows that our bounds are tight. We then move on to parametric quantum pumps, which make it possible to move a welldefined amount of carriers from one reservoir to another in a given cycle time. Such devices can be realized, for instance, with tunable-barrier quantum dots [70,71], and, owing to their high accuracy, are promising candidates for experimentally accessible quantum representations of the ampere [20-22]. Here, we show that, in the slow-driving regime, the energy that is required to move a given amount of carriers is subject to a fundamental lower bound, which depends only on the cycle time. We further derive an explicit optimization principle for adiabatic quantum pumps by connecting our theory with the geometric approach to parametric pumping [72-78] and the notion of thermodynamic length [79-83]. As a third application of our theory, we derive a universal tradeoff relation between the efficiency and the power consumption of adiabatic quantum motors, that is, devices that convert an electric current into motive power of mesoscopic mechanical objects like nanopaddle wheels or conveyor belts [23-26].

\section{A. Quantum generator}

The setup of Fig. 2 provides a simple realization of a quantum generator. The frozen scattering amplitudes for this system are
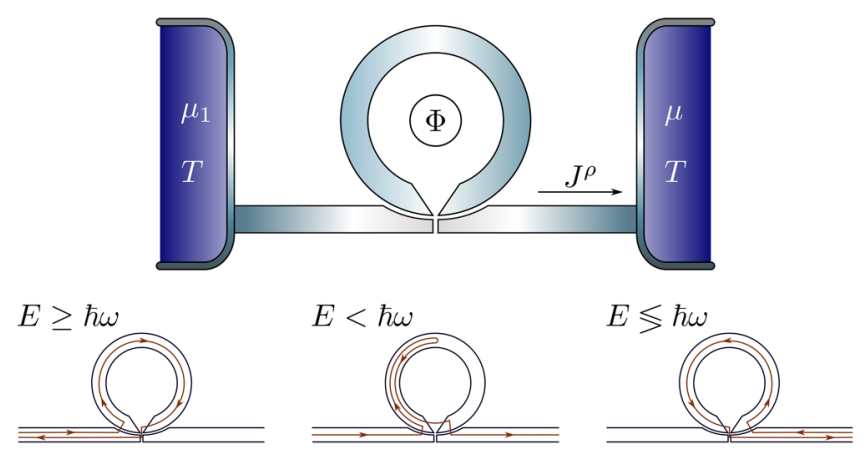

FIG. 2. Quantum generator. A mesoscopic ring is connected to two leads via a four-way beam splitter such that, without a magnetic flux, all incoming carriers are reflected after passing through the ring. The current $J^{\rho}$ is generated by changing the magnetic flux $\Phi$ such that clockwise-moving carriers are decelerated by the emerging electromagnetic force around the ring. As a result, incoming carriers from the left are reflected only if their energy $E$ is above the threshold $\hbar \omega$, which is required to pass through the entire loop; carriers with $E<\hbar \omega$ are flipped around and transmitted to the right. At the same time, carriers coming from the right are accelerated and thus reflected regardless of their energy. 


$$
\begin{aligned}
& \mathcal{S}_{E, \phi}^{11}=e^{i\left(\chi_{E}+\phi\right)}, \quad \mathcal{S}_{E, \phi}^{12}=\mathcal{S}_{E, \phi}^{21}=0, \\
& \mathcal{S}_{E, \phi}^{22}=e^{i\left(\chi_{E}-\phi\right)},
\end{aligned}
$$

where the irrelevant dynamical phase $\chi_{E}$ is determined by the circumference of the loop and the Aharonov-Bohm phase $\phi \equiv e \Phi / \hbar c$ plays the role of an external control parameter; here, $\Phi$ is the tunable magnetic flux through the ring, $e$ is the carrier charge, and $c$ the speed of light [84]. We use the reservoir on the right as a reference. Hence, the chemical affinity is $F_{1}^{\rho} \equiv F^{\rho}$ and the electric current $J_{1}^{\rho} \equiv$ $J^{\rho}$ flows from left to right. For a linearly increasing flux, i.e., for $\phi_{t} \equiv \omega t$, the kinetic coefficients are

$$
\begin{aligned}
L^{\rho \rho} & =0, \quad L^{\rho \omega}=-L^{\omega \rho}=\frac{T \varphi}{h(1+\varphi)}, \\
L^{\omega \omega} & =\frac{T \varphi}{h(1+\varphi)},
\end{aligned}
$$

according to the formulas (22), where $\varphi=\exp [\mu / T]$ is the fugacity of the reservoir on the right. The electric current and the rate of entropy production are thus given by $J^{\rho}=L^{\rho \omega} F^{\omega}$ and $\sigma=L^{\omega \omega}\left(F^{\omega}\right)^{2}$. Upon recalling the expressions (46) for the coefficient $K^{\rho \rho}$, it is now straightforward to verify that the bound (45) is saturated; that is, $\sigma=\left(J^{\rho}\right)^{2} / 2 K^{\rho \rho}$ for any $F^{\rho}$ and $F^{\omega}$. This result shows that our bounds are tight.

On the microscopic level, the saturation of the bound (45) is a consequence of the working mechanism of the quantum generator, which is described in Fig. 2. Every transmitted carrier leads to the net dissipation of one quantum of energy $\hbar \omega$. As a result, the irreversible part of the photon current, $J_{\text {irr }}^{\omega}=L^{\omega \omega} F^{\omega}$, is equal to the electric current $J^{\rho}$ and proportional to the rate of entropy production $\sigma=F^{\omega} J_{\text {irr }}^{\omega}$.

By contrast, the reversible photon current, $J_{\text {rev }}^{\omega}=L^{\omega \rho} F^{\rho}$, is decoupled from the dissipation rate $\sigma$, which is independent of the chemical affinity $F^{\rho}$. In particular, for $F^{\omega}=$ 0 and $F^{\rho} \neq 0$, we have $\sigma=0$ and $J^{\omega}=J_{\text {rev }}^{\omega} \neq 0$. This observation does not imply the occurrence of dissipationless transport, since the electric current vanishes for $F^{\omega}=0$. It shows, however, that no general bound of the form Eq. (45) exists for the photon current.

\section{B. Parametric quantum pumps}

\section{Performance bound}

A parametric quantum pump can be described as a twoterminal conductor, whose potential landscape is changed periodically to generate a flow of carriers between two reservoirs with the same chemical potential and temperature. One pumping cycle requires the energy input $U \equiv$ $\mathcal{T} \Pi_{\mathrm{ac}}$ and moves the amount of carriers $\mathcal{Q} \equiv \mathcal{T} J^{\rho}$ from the first reservoir to the second; see Fig. 3. Our bound (45) implies that, in the slow-driving regime, these two figures are connected by the trade-off relation,
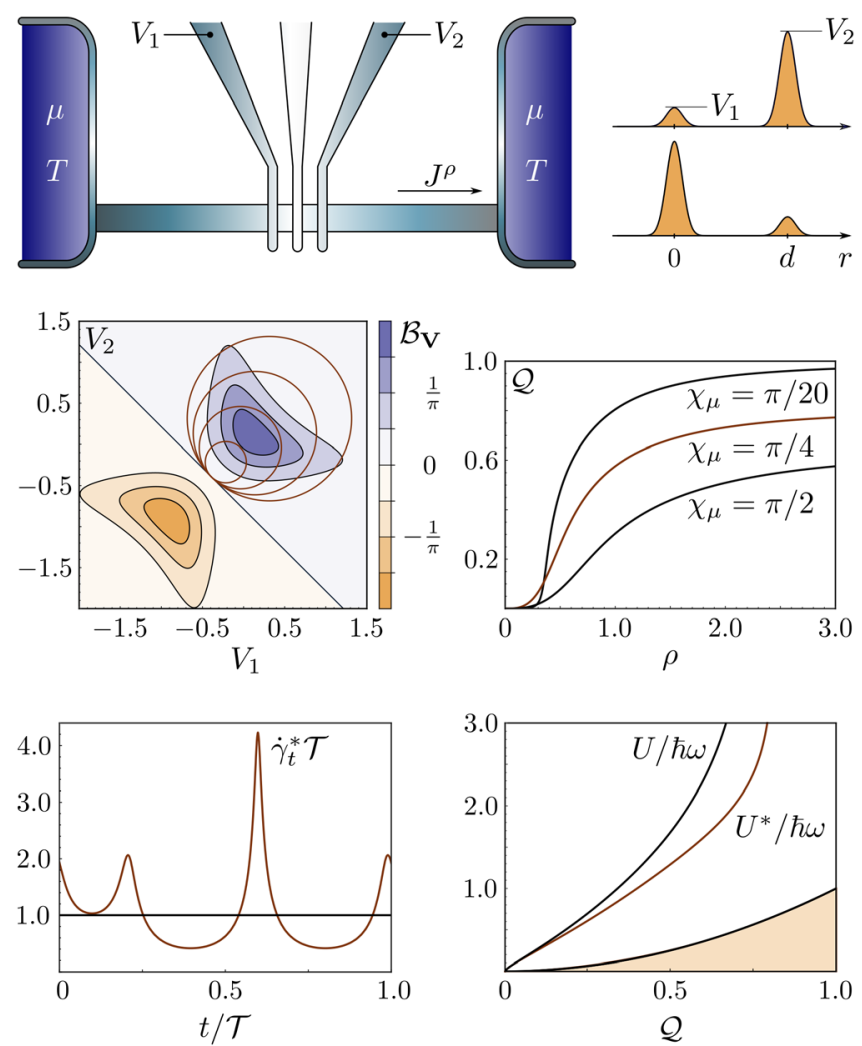

FIG. 3. Parametric quantum pump. Top: sketch of a generic setup. A narrow conductor connects two reservoirs with the same chemical potential and temperature. Periodically changing the gate voltages $V_{1}$ and $V_{2}$ creates two oscillating potential barriers driving the pump current $J^{\rho}$. Middle: the left plot shows the Berry curvature $\mathcal{B}_{\mathbf{V}}$ for $\chi_{\mu}=\pi / 4$. Circles indicate the control path that is determined by the protocols (61) for $\rho=1 / 4,1 / 2,3 / 4,1$. As the path expands into the positive peak of $\mathcal{B}_{\mathbf{V}}$, the pump flux $\mathcal{Q}$ first increases sharply and then approaches a limit value, which depends on the parameter $\chi_{\mu}$. Bottom: the left-hand panel shows the optimal driving speed $\dot{\gamma}_{t}^{*}$ for $\chi_{\mu}=\pi / 4$ and $\rho=1$. The horizontal line corresponds to constant speed. On the right, the energy uptake is plotted against the pump flux for constant and optimal driving speed $U$ and $U^{*}$, respectively, where $\chi_{\mu}=$ $\pi / 4$ and $\rho$ varies between 0 and 5. The shaded area indicates the bound (49).

$$
U \geq \frac{T \mathcal{Q}^{2}}{2 \mathcal{T} K^{\rho \rho}} \geq \hbar \omega \mathcal{Q}^{2}
$$

where we have used that $U=\mathcal{T} T \sigma$ and the second inequality follows from Eq. (46a). This result is quite remarkable as it puts a universal lower bound on the energy that must be provided to generate a given pump flux $\mathcal{Q}$ in a given cycle time $\mathcal{T}$. Hence, Eq. (49) makes it possible to estimate the energy consumption of a quantum pump, even in situations, where only the flux $\mathcal{Q}$ can be measured and the scattering amplitudes of the sample are unknown. 


\section{Geometry and optimal driving speed}

Finding optimal driving protocols for a quantum pump is a difficult task, which typically requires the solution of involved variational problems. In the slow-driving regime, it is, however, possible to derive a universal optimization principle for the parametrization $\gamma$ of the closed path $\Gamma$ that is mapped out by the vector of control parameters $\mathbf{V}_{t}$ during the cycle. To this end, we recall that, by using Eq. (22c), the pump flux can be written as a line integral in the space of control parameters [72],

$$
\mathcal{Q}=\mathcal{T} L^{\rho w} F^{\omega}=\oint_{\Gamma} \sum_{j} \mathcal{A}_{\mathbf{V}}^{j} d V_{j}
$$

which proves that $\mathcal{Q}$ is independent of the parametrization of $\Gamma$. The objects

$$
\mathcal{A}_{\mathbf{V}}^{j} \equiv \frac{1}{2 \pi T} \int_{0}^{\infty} d E \sum_{\beta} \operatorname{Im}\left[S_{E, \mathbf{V}}^{1 \beta *} \partial_{V_{j}} S_{E, \mathbf{V}}^{1 \beta}\right] f_{E}^{\prime}
$$

are thereby considered as the components of a vector field $\mathcal{A}_{\mathbf{v}}$, which, in analogy to the theory of geometric phases, is called the Berry potential [85]. Second, the input $U$ can be expressed in the form

$$
U=T \mathcal{T} L^{\omega \omega}\left(F^{\omega}\right)^{2}=h \int_{0}^{\mathcal{T}} d t \sum_{i j} g_{\mathbf{V}_{t}}^{i j} \dot{V}_{i, t} \dot{V}_{j, t}
$$

where we have used Eq. (22d) and introduced the thermodynamic metric [86],

$g_{\mathbf{V}}^{i j} \equiv \frac{1}{8 \pi^{2} T} \int_{0}^{\infty} d E \sum_{\alpha \beta} \operatorname{Re}\left[\left(\partial_{V_{i}} S_{E, \mathbf{V}}^{\alpha \beta}\right)\left(\partial_{V_{j}} S_{E, \mathbf{V}}^{\alpha \beta *}\right)\right] f_{E}^{\prime}$.

The optimal parametrization $\gamma_{t}^{*}$ of the path $\Gamma$, which minimizes the input $U$, can be determined from the objective functional,

$$
O\left[\dot{\hat{\gamma}}_{t}\right] \equiv \int_{0}^{\mathcal{T}} d t\left(\sum_{i j} g_{\mathbf{V}_{t}}^{i j} \dot{V}_{i, t} \dot{V}_{j, t} / \dot{\hat{\gamma}}_{t}-\Lambda \dot{\hat{\gamma}}_{t}\right)
$$

where $\Lambda$ is a Lagrange multiplier accounting for the boundary condition $\gamma_{\mathcal{T}}-\gamma_{0}=\mathcal{T}$ and $\dot{\hat{\gamma}}_{t}$ is the derivative of the inverse function $\hat{\gamma}_{t}$ of $\gamma_{t}$ [87]. Solving the EulerLagrange equation for this functional yields the condition

$$
t=\frac{\mathcal{T}}{\mathcal{L}} \int_{0}^{\gamma_{t}^{*}} d s \sqrt{\sum_{i j} g_{\mathbf{V}_{s}}^{i j} \dot{V}_{i, s} \dot{V}_{j, s}},
$$

where

$$
\mathcal{L} \equiv \oint_{\Gamma} \sqrt{\sum_{i j} g_{\mathbf{V}}^{i j} d V_{i} d V_{j}}
$$

denotes the thermodynamic length of $\Gamma$. Replacing the protocols $\mathbf{V}_{t}$ with $\mathbf{V}_{\gamma_{t}^{*}}$ minimizes the energy consumption of the pump without changing its flux $\mathcal{Q}$, which depends only on the path $\Gamma$. Inserting the minimal energy uptake $U^{*}=$ $\hbar \omega \mathcal{L}^{2}$ into Eq. (49) yields the remarkably simple relation,

$$
\mathcal{L} \geq|\mathcal{Q}|,
$$

between thermodynamic length and pump flux, which connects our thermodynamic bounds with the geometric theory of slowly driven quantum pumps.

\section{Tunable-barrier pump}

We now consider a simple model of a quantum pump, where the potential inside the conductor consists of two $\delta$-barriers with dimensionless strengths $V_{1}$ and $V_{2}$. The single-particle Hamiltonian of this system reads

$$
H_{\mathbf{V}}=\frac{p^{2}}{2 M}+\frac{\hbar^{2} V_{1}}{M d} \delta_{r}+\frac{\hbar^{2} V_{2}}{M d} \delta_{r-d}
$$

where $\mathbf{V} \equiv\left(V_{1}, V_{2}\right), p$ and $r$ are the momentum and position of the carrier, $d$ denotes the distance between the two barriers, and $M$ the carrier mass; see Fig. 3. The corresponding frozen scattering amplitudes are [6]

$\mathcal{S}_{E, \mathbf{V}}^{12}=\mathcal{S}_{E, \mathbf{V}}^{21}=\mathcal{Z}_{E, \mathbf{v}} \chi_{E}^{2} e^{i \chi_{E}}$,

$\mathcal{S}_{E, \mathbf{V}}^{11}=\mathcal{Z}_{E, \mathbf{V}}\left(V_{1}\left(V_{2}-i \chi_{E}\right)-V_{2}\left(V_{1}+i \chi_{E}\right) e^{2 i \chi_{E}}\right)$,

$\mathcal{S}_{E, \mathbf{V}}^{22}=\mathcal{Z}_{E, \mathbf{V}}\left(V_{2}\left(V_{1}-i \chi_{E}\right)-V_{1}\left(V_{2}+i \chi_{E}\right) e^{2 i \chi_{E}}\right)$,

with $\mathcal{Z}_{E, \mathbf{V}} \equiv 1 /\left(V_{1} V_{2} e^{2 i \chi_{E}}-\left(V_{1}-i \chi_{E}\right)\left(V_{2}-i \chi_{E}\right)\right)$ and $\chi_{E} \equiv d \sqrt{2 M E} / \hbar$. In the following, we focus on the lowtemperature limit, where the function $f_{E}^{\prime}$ is sharply peaked around $E=\mu$ and can therefore be replaced with $T \delta_{E-\mu}$ in Eqs. (50) and (53).

To find a suitable control path, we recall that the expression (50) for the pump flux can be rewritten as an area integral with the help of Stokes' theorem [72],

$$
\mathcal{Q}=\int_{S_{\Gamma}} d S \mathcal{B}_{\mathbf{V}}
$$

where $\mathcal{B}_{\mathbf{V}} \equiv \partial_{V_{1}} \mathcal{A}_{\mathbf{V}}^{2}-\partial_{V_{2}} \mathcal{A}_{\mathbf{V}}^{1}$ is the Berry curvature corresponding to the potential $\mathcal{A}_{\mathbf{V}}$ and $S_{\Gamma}$ denotes the area encircled by $\Gamma$. As shown in Fig. 3, the function $\mathcal{B}_{\mathbf{V}}$ features two antisymmetric peaks. Hence, to generate a large flux $\mathcal{Q}$, the path $\Gamma$ has to cover the positive peak while avoiding the negative one. This condition is met by the circles with the parametrization, 
$V_{1, t}=V_{0}-\rho \cos [\omega t], \quad V_{2, t}=V_{0}-\rho \sin [\omega t]$,

where $V_{0} \equiv \rho / \sqrt{2}-\chi_{\mu} \cot \left[\chi_{\mu}\right] / 2$ and the radius $\rho$ determines the amplitude of the driving.

Using the protocols (61), we numerically calculate the flux $\mathcal{Q}$, the input $U$, the optimal driving speed $\dot{\gamma}^{*}$, and the minimized input $U^{*}$. The results of these calculations are plotted in Fig. 3. Two observations stand out. First, the energy consumption of the pump can indeed be significantly reduced by optimizing the driving speed. Second, our bound (49) underestimates the energy uptake by at least a factor of 6 for constant and at least a factor of 4 for optimal driving speed. Microscopically, the deviations arise from idle scattering events, where carriers pick up energy from the external driving without contributing to the pump flux. This effect becomes more and more dominant as the amplitude $\rho$ of the potential modulations increases. Still, at least for moderate amplitudes, our bound (49) predicts the correct order of magnitude for the energy uptake of the device.

\section{Adiabatic quantum motors \\ 1. Bound on efficiency}

A quantum motor can be described in terms of two components: a mesoscopic conductor hosting an electric current $J^{\rho}$ between two reservoirs with the same temperature but different chemical potentials and a mechanical rotor that couples to the dynamics of the carriers [24]; see Fig. 4. Provided that the rotor is much heavier than the carriers, it can be treated as a classical degree of freedom, which creates a slowly and periodically changing potential inside the conductor [88]. In this Born-Oppenheimer picture, the motive power of the rotor, that is, the output of the motor, is given by $\Pi_{\mathrm{m}} \equiv-\Pi_{\mathrm{ac}}=-T F^{\omega} J^{\omega}$. The electric power $\Pi_{\mathrm{el}}=T F^{\rho} J^{\rho}$ is the input of the motor and its efficiency is defined as

$$
\eta_{\mathrm{m}} \equiv \Pi_{\mathrm{m}} / \Pi_{\mathrm{el}} \leq 1
$$

for $\Pi_{\mathrm{m}}>0$. The upper bound 1 thereby follows from the second law, which requires $\sigma=\Pi_{\mathrm{el}} / T-\Pi_{\mathrm{m}} / T \geq 0$.

Going beyond this trivial result, our bound (45) implies that the efficiency and the input of the device are connected by the universal relation,

$$
\eta_{\mathrm{m}} \leq 1-\frac{\Pi_{\mathrm{el}}}{2 T K^{\rho \rho}\left(F^{\rho}\right)^{2}} \leq 1-\frac{h \Pi_{\mathrm{el}}}{\Delta \mu^{2}}=1-\frac{h J^{\rho}}{\Delta \mu},
$$

where the second inequality follows from Eq. (46a). Depending only on the electric current $J^{\rho}$ and the chemical potential bias $\Delta \mu=\mu_{1}-\mu$, both of which are generally easy to access in experiments, the bound (63) has a key practical implication: it makes it possible to put a nontrivial upper limit on the efficiency of an adiabatic quantum motor
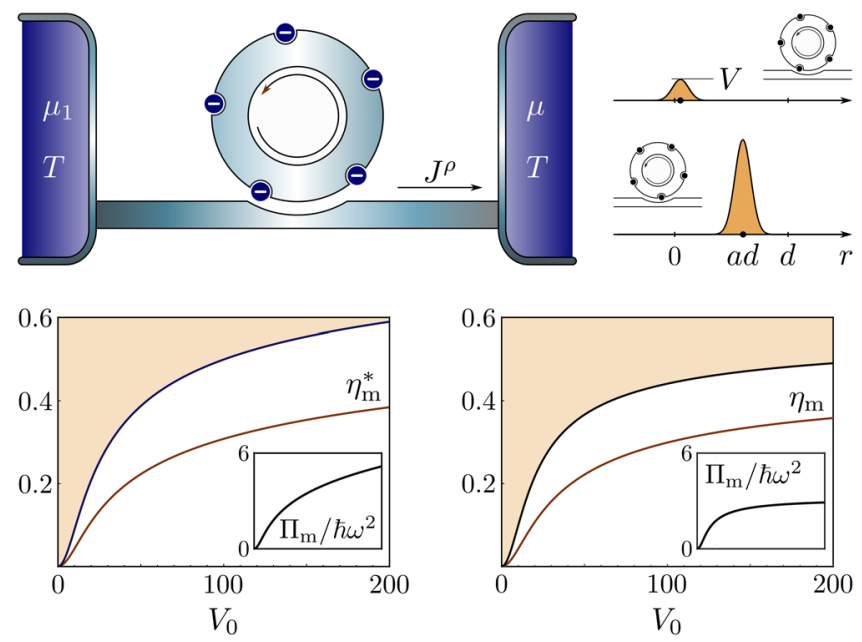

FIG. 4. Quantum paddle wheel motor. Top: setup. A narrow coherent conductor, which is placed between two isothermal reservoirs with chemical potentials $\mu_{1}$ and $\mu<\mu_{1}$, is in contact with a mesoscopic wheel, the rotor, which carries negative charges on its surface acting as electrostatic paddles. The electric current $J^{\rho}$ pushes the potential barrier that is created by a passing charge to the right, thus driving the rotation of the wheel. Bottom: the left-hand plot shows the maximum efficiency (69) of the motor as a function of the strength of the barrier $V_{0}$ with the shaded area corresponding to the bound (63). The inset shows the generated motive power over the same range of $V_{0}$ as in the main panel. For comparison, the efficiency and the motive power are plotted on the right-hand side for fixed bias $v=10$. For all plots, we have set $\chi_{\mu}=4 \pi$.

without measuring the motive power of the rotor or invoking a specific model.

\section{Paddle wheel motor}

To test the accuracy of the bound (63), we consider a simple quantum motor, which consists of a rotating nanopaddle wheel creating a sliding $\delta$-barrier inside a narrow conductor; see Fig. 4. The single-particle Hamiltonian of this model is

$$
H_{\mathbf{V}}=\frac{p^{2}}{2 M}+\frac{\hbar^{2} V}{M d} \delta_{r-d a}
$$

and the frozen scattering amplitudes are given by

$$
\begin{aligned}
& \mathcal{S}_{E, \mathbf{V}}^{12}=\mathcal{S}_{E, \mathbf{V}}^{21}=i \chi_{E} e^{i \chi_{E}} /\left(i \chi_{E}-V\right), \\
& \mathcal{S}_{E, \mathbf{V}}^{11}=V e^{2 i a \chi_{E}} /\left(i \chi_{E}-V\right), \\
& \mathcal{S}_{E, \mathbf{V}}^{22}=V e^{2 i(1-a) \chi_{E}} /\left(i \chi_{E}-V\right),
\end{aligned}
$$

with $\mathbf{V} \equiv(V, a)$ and $\chi_{E} \equiv d \sqrt{2 M E} / \hbar$. Here, $d$ denotes the length of the contact region between the conductor and the paddle wheel and the dimensionless control parameters $V$ and $a \in[0,1]$ correspond to the strength and the position of 
the barrier. In the low-temperature limit, the electric and the motive power are given by

$$
\begin{aligned}
& \Pi_{\mathrm{el}} / \hbar \omega^{2}=\bar{L}^{\rho \rho} v^{2}+\bar{L}^{\rho \omega} v, \\
& \Pi_{\mathrm{m}} / \hbar \omega^{2}=-\bar{L}^{\omega \rho} v-\bar{L}^{\omega \omega},
\end{aligned}
$$

where $v \equiv \Delta \mu / \hbar \omega$ and we have introduced the rescaled kinetic coefficients:

$$
\begin{aligned}
\bar{L}^{\rho \rho} & =\frac{1}{2 \pi}\left\langle\left\langle\left|\mathcal{S}_{\mu, \mathbf{V}}^{12}\right|^{2}\right\rangle\right\rangle, \\
\bar{L}^{\rho \omega} & =-\bar{L}^{\omega \rho}=\frac{1}{2 \pi \omega} \sum_{\beta} \operatorname{Im}\left[\left\langle\mathcal{S}_{\mu, \mathbf{V}}^{\beta 1} \dot{\mathcal{S}}_{\mu, \mathbf{V}}^{\beta 1 *}\right\rangle\right], \\
\bar{L}^{\omega \omega} & =\frac{1}{4 \pi \omega^{2}} \sum_{\alpha \beta}\left\langle\left\langle\left|\dot{\mathcal{S}}_{\mu, \mathbf{V}}^{\alpha \beta}\right|^{2}\right\rangle\right\rangle .
\end{aligned}
$$

We now calculate $\Pi_{\mathrm{el}}$ and $\Pi_{\mathrm{m}}$ for the protocols

$$
V_{t}=V_{0} \sin ^{2}[\omega t / 2] \text { and } a_{t}=t / \mathcal{T} \quad \bmod 1,
$$

which mimic the motion of the paddle wheel, assuming that its radius is much larger than $d$. The bias $v$ is fixed by maximizing the efficiency of the motor (62). That is, we set $v=v^{*} \equiv \bar{L}^{\omega \omega}\left(1+\sqrt{Z_{\mathrm{m}}+1}\right) / \bar{L}^{\rho \omega}$, whereby the efficiency becomes

$$
\eta_{\mathrm{m}}^{*}=\frac{\sqrt{Z_{\mathrm{m}}+1}-1}{\sqrt{Z_{\mathrm{m}}+1}+1},
$$

with $Z_{\mathrm{m}} \equiv\left(\bar{L}^{\rho \omega}\right)^{2} / \bar{L}^{\rho \rho} \bar{L}^{\omega \omega}$ playing the role of a figure of merit. Our results are plotted in Fig. 4. For $V_{0} \lesssim 10$, the bound (63) overestimates the efficiency of the paddle wheel motor by about a factor of 2 . As $V_{0}$ increases, the deviation decays to approximately a factor of $3 / 2$, since fewer carriers tunnel through the moving barrier without transmitting energy to the paddle wheel. The same qualitative behavior is observed if the bias $v$ is fixed independently of the kinetic coefficients.

\section{FAR FROM EQUILIBRIUM}

Going beyond the first and the second law, our bounds (43) and (45) provide strong universal constraints on currents in periodically driven coherent conductors. They were derived within the framework of linear response theory, which describes the arguably most relevant regime of operation of mesoscopic devices. Still, the question remains whether similar bounds apply also far from equilibrium. In this section, we show how such a generalization of our theory can be achieved. We first derive a family of new bounds on currents in coherent conductors that hold for arbitrary driving frequencies and thermal gradients. We then discuss the interpretation of these bounds in the context of thermodynamic uncertainty relations $[89,90]$ and show how they can be used for thermodynamic inference. To illustrate the general picture, we revisit the quantum generator of Sec. VA, whose Floquet scattering amplitudes can be calculated exactly for arbitrary driving frequencies. We then put our results in the context of recent developments and conclude this section by summarizing the main implications of our theory for systems without time-dependent driving.

\section{A. Derivation}

To extend the approach of Sec. IV A into the nonlinear regime, we consider the quadratic form:

$\Xi_{\psi} \equiv \sigma+\psi \sum_{\alpha} \sum_{x} J_{\alpha}^{x} G_{\alpha}^{x}+\psi \sum_{\alpha} \sum_{x y} \hat{K}_{\alpha}^{x y} G_{\alpha}^{x} G_{\alpha}^{y}$.

Here, $\psi$ is a yet undetermined positive number and the coefficients $\hat{K}_{\alpha}^{x y}$ are defined as

$$
\begin{aligned}
\hat{K}_{\alpha}^{x y} \equiv & \frac{1}{4 h} \int_{0}^{\infty} d E \sum_{\beta} \sum_{n} \zeta_{E n}^{x, \alpha} \zeta_{E n}^{y, \alpha}\left|\hat{S}_{E n, E}^{\alpha \beta}\right|^{2} \\
& \times\left(f_{E n}^{\alpha}\left(1-f_{E}^{\beta}\right)+f_{E}^{\beta}\left(1-f_{E n}^{\alpha}\right)\right),
\end{aligned}
$$

with $\zeta_{E}^{\rho, \alpha} \equiv 1, \zeta_{E}^{q, \alpha} \equiv E-\mu_{\alpha}$, and the modified Floquet scattering amplitudes,

$$
\hat{S}_{E n, E}^{\alpha \beta} \equiv\left(1-\delta_{n 0} \delta_{\alpha \beta}\right) S_{E n, E}^{\alpha \beta} .
$$

This ansatz is essentially found by inspection but can be motivated a posteriori, as we shall see in Sec. VIB.

We now recall Eqs. (4), (13), and (14) and use the unitarity conditions (8) to express the thermochemical currents and the rate of entropy production as

$$
\begin{aligned}
J_{\alpha}^{x}= & \left.\frac{1}{h} \int_{0}^{\infty} d E \sum_{\beta} \sum_{n} \zeta_{E n}^{x, \alpha}||_{E n, E}^{\alpha \beta}\right|^{2}\left(f_{E n}^{\alpha}-f_{E}^{\beta}\right), \\
\sigma= & \frac{1}{h} \int_{0}^{\infty} d E \sum_{\alpha \beta} \sum_{n}\left|\hat{S}_{E n, E}^{\alpha \beta}\right|^{2} \\
& \times\left(\left(\nu_{E}^{\beta}-\nu_{E n}^{\alpha}\right) f_{E}^{\beta}-g_{E}^{\beta}+g_{E n}^{\alpha}\right),
\end{aligned}
$$

with $\nu_{E}^{\alpha} \equiv\left(\mu_{\alpha}-E\right) / T_{\alpha}$ and $g_{E}^{\alpha} \equiv \nu_{E}^{\alpha}-\ln \left[f_{E}^{\alpha}\right]$. Inserting these expressions and the definition (71) into Eq. (70) gives

$$
\Xi_{\psi}=\frac{1}{h} \int_{0}^{\infty} d E \sum_{\alpha \beta} \sum_{n}\left|\hat{S}_{E n, E}^{\alpha \beta}\right|^{2} \Xi_{\psi, n, E}^{\alpha \beta},
$$

where 


$$
\begin{aligned}
\Xi_{\psi, n, E}^{\alpha \beta} \equiv & \left(\nu_{E}^{\beta}-\nu_{E n}^{\alpha}\right) f_{E}^{\beta}-g_{E}^{\beta}+g_{E n}^{\alpha}+2 \psi X_{E n}^{\alpha}\left(f_{E n}^{\alpha}-f_{E}^{\beta}\right) \\
& +\psi\left(X_{E n}^{\alpha}\right)^{2}\left(f_{E n}^{\alpha}\left(1-f_{E}^{\beta}\right)+f_{E}^{\beta}\left(1-f_{E n}^{\alpha}\right)\right)
\end{aligned}
$$

is a quadratic form in the variables $X_{E}^{\alpha} \equiv \sum_{x} \zeta_{E}^{x, \alpha} G_{\alpha}^{x} / 2$. As has been shown in Ref. [47], this quadratic form is positive semidefinite if

$\psi \leq \psi^{*} \equiv \min _{z \in \mathbb{R}} \frac{\left(1-e^{z}+z e^{z}\right)\left(e^{z}+1\right)}{\left(e^{z}-1\right)^{2}} \simeq 0.89612$.

Consequently, we have $\Xi_{\psi^{*}} \geq 0$ for any choice of the auxiliary variables $G_{\alpha}^{x}$.

From here, we can proceed as in Sec. IV A. Minimizing $\Xi_{\psi^{*}}$, first with respect to the $\left\{G_{\alpha}^{\rho}\right\}$ and then with respect to the $\left\{G_{\alpha}^{q}\right\}$ while setting the respectively remaining auxiliary variables to zero, yields the cumulative bound,

$$
\sigma \geq \psi^{*} \sum_{\alpha} \frac{\left(J_{\alpha}^{x}\right)^{2}}{4 \hat{K}_{\alpha}^{x x}}
$$

which can be regarded as the counterpart of Eq. (43). For the individual matter and heat currents, we obtain the bounds,

$$
\begin{aligned}
& \sigma \geq \frac{\psi^{*}\left(1+\theta_{\alpha}^{\rho}\right)}{4 \hat{K}_{\alpha}^{\rho \rho}}\left(J_{\alpha}^{\rho}\right)^{2}, \\
& \sigma \geq \frac{\psi^{*}}{4 \hat{K}_{\alpha}^{q q}}\left(\left(J_{\alpha}^{q}\right)^{2}+\left(J_{\alpha}^{q}+\Pi_{\mathrm{ac}}+\Pi_{\mathrm{el}}\right)^{2} \theta_{\alpha}^{q}\right),
\end{aligned}
$$

with $\theta_{\alpha}^{x} \equiv \hat{K}_{\alpha}^{x x} / \sum_{\beta \neq \alpha} \hat{K}_{\beta}^{x x} \geq 0$, which can be derived from Eq. (77) by repeating the steps that led from Eq. (43) to Eq. (45); for the heat currents, the conservation law $\sum_{\alpha} J_{\alpha}^{q}=0$, which holds only in linear response, must thereby be replaced with the first law (12).

We note that, in linear response, the bounds (78) are weaker than our previous bound (45) by a factor $\psi^{*}$. Specifically, upon neglecting third-order corrections in the affinities, Eqs. (78) imply

$$
\sigma \geq \frac{\psi^{*}\left(1+\left.\theta_{\alpha}^{x}\right|_{\mathrm{eq}}\right)}{\left.4 \hat{K}_{\alpha}^{x x}\right|_{\mathrm{eq}}}\left(J_{\alpha}^{x}\right)^{2} \geq \frac{\psi^{*} N}{4 K^{x x}(N-1)}\left(J_{\alpha}^{x}\right)^{2},
$$

where the second inequality follows by noting that

$$
\left.\hat{K}_{\alpha}^{x x}\right|_{\mathrm{eq}}=K^{x x}-\frac{1}{2 h} \int_{0}^{\infty} d E\left(\zeta_{E}^{x}\right)^{2} \mid\left\langle\left.\left\langle\mathcal{S}_{E, \mathbf{V}}^{\alpha \alpha}\right\rangle\right|^{2} f_{E}^{\prime} \leq K^{x x},\right.
$$

and, therefore,

$$
\frac{1+\left.\theta_{\alpha}^{x}\right|_{\mathrm{eq}}}{\left.\hat{K}_{\alpha}^{x x}\right|_{\mathrm{eq}}}=\frac{1}{\left.\hat{K}_{\alpha}^{x x}\right|_{\mathrm{eq}}}+\frac{1}{\left.\sum_{\beta \neq \alpha} \hat{K}_{\beta}^{x x}\right|_{\mathrm{eq}}} \geq \frac{N}{K^{x x}(N-1)} .
$$

This observation shows that the bounds (78) cannot be saturated close to equilibrium.

\section{B. Thermodynamic uncertainty relations}

A thermodynamic uncertainty relation describes the trade-off between precision and entropy production in a given class of thermodynamic processes [89,90]. For classical matter transport in multiterminal systems without time-dependent driving, for example, the relation

$$
\sigma\left(\epsilon_{\alpha}^{\rho}\right)^{2} \geq \psi^{*}
$$

has been derived in Ref. [47]. The reduced zero-frequency noise, or squared relative uncertainty, $\left(\epsilon_{\alpha}^{\rho}\right)^{2} \equiv P_{\alpha \alpha}^{\rho \rho} /\left(J_{\alpha}^{\rho}\right)^{2}$, thereby provides a measure for the accuracy at which a given amount of particles is extracted from the reservoir $\alpha$ in a given time. As we show in the following, our bounds (78) make it possible to extend this relation into the quantum regime and to include heat currents as well as periodic driving.

We first recall that, according to the formulas (6) and (7), the diagonal thermal and the shot noise of the thermochemical currents are given by

$$
\begin{aligned}
D_{\alpha \alpha}^{x x}= & \sum_{u v} c_{\alpha}^{x u} c_{\alpha}^{x v} D_{\alpha \alpha}^{u v} \\
= & \frac{1}{h} \int_{0}^{\infty} d E \sum_{\beta} \sum_{n}\left(\left(\zeta_{E n}^{x, \alpha}\right)^{2}\left|S_{E n, E}^{\alpha \beta}\right|^{2}\left(f_{E n}^{\prime \alpha}+f_{E}^{\prime \beta}\right)\right. \\
& \left.-2 \delta_{\alpha \beta} \zeta_{E n}^{x, \alpha} \zeta_{E}^{x, \alpha}\left|S_{E n, E}^{\alpha \alpha}\right|^{2} f_{E}^{\prime \alpha}\right),
\end{aligned}
$$

$$
\begin{aligned}
R_{\alpha \alpha}^{x x} & =\sum_{u v} c_{\alpha}^{x u} c_{\alpha}^{x v} R_{\alpha \alpha}^{u v} \\
& =\frac{1}{2 h} \int_{0}^{\infty} d E \sum_{\gamma \delta} \sum_{n}\left|\sum_{u} c_{\alpha}^{x u} C_{n, E}^{u \alpha, \gamma \delta}\right|^{2},
\end{aligned}
$$

with $c_{\alpha}^{\rho u} \equiv \delta_{u \rho}, c_{\alpha}^{q u} \equiv \delta_{u \varepsilon}-\mu_{\alpha} \delta_{u \rho}$, and $f_{E}^{\prime \alpha} \equiv f_{E}^{\alpha}\left(1-f_{E}^{\alpha}\right)$. Next, comparing Eq. (83a) with Eq. (71) shows that the coefficients $\hat{K}_{\alpha}^{x x}$ can be decomposed as

$$
4 \hat{K}_{\alpha}^{x x}=D_{\alpha \alpha}^{x x}+\Psi_{\alpha}^{x x}+\Omega_{\alpha}^{x x} .
$$

The Fermi correction,

$\Psi_{\alpha}^{x x} \equiv \frac{1}{h} \int_{0}^{\infty} d E \sum_{\beta} \sum_{n}\left(\zeta_{E n}^{x, \alpha}\right)^{2}\left|\hat{S}_{E n, E}^{\alpha \beta}\right|^{2}\left(f_{E n}^{\alpha}-f_{E}^{\beta}\right)^{2}$,

thereby accounts for Pauli blocking between incoming and outgoing carriers; it vanishes in equilibrium and in the quasiclassical limit, where second-order terms in the fugacities $\varphi_{\alpha} \equiv \exp \left[\mu_{\alpha} / T_{\alpha}\right]$ can be neglected and the exclusion principle becomes irrelevant [29]. The second correction in Eq. (84), 


$$
\Omega_{\alpha}^{x x} \equiv \frac{2}{h} \int_{0}^{\infty} d E \sum_{n} \zeta_{E n}^{x, \alpha} \zeta_{E}^{x, \alpha}\left|\hat{S}_{E n, E}^{\alpha \alpha}\right|^{2} f_{E}^{\prime \alpha}
$$

arises from inelastic reflections of carriers at the sample and vanishes if the external driving is turned off, i.e., if $S_{E n, E}^{\alpha \alpha}=\delta_{n 0} S_{E}^{\alpha \alpha}$ and thus $\hat{S}_{E n, E}^{\alpha \alpha}=0$. Finally, since the shot noise (83b) is non-negative, Eq. (84) implies that $\hat{K}_{\alpha}^{x x} \leq P_{\alpha \alpha}^{x x}+\Psi_{\alpha}^{x x}+\Omega_{\alpha}^{x x}$, where $P_{\alpha \alpha}^{x x}=D_{\alpha \alpha}^{x x}+R_{\alpha \alpha}^{x x}$ is the zero-frequency noise of the thermochemical currents. Inserting this bound into Eqs. (78) and setting $\theta_{\alpha}^{x}=0$ yields the thermodynamic uncertainty relation,

$$
\sigma\left(\epsilon_{\alpha}^{x}\right)^{2} \geq \frac{\psi^{*}}{1+\Psi_{\alpha}^{x x} / P_{\alpha \alpha}^{x x}+\Omega_{\alpha}^{x x} / P_{\alpha \alpha}^{x x}},
$$

where $\left(\epsilon_{\alpha}^{x}\right)^{2} \equiv P_{\alpha \alpha}^{x x} /\left(J_{\alpha}^{x}\right)^{2}$.

This result can be interpreted as follows. The corrections $\Psi_{\alpha}^{\rho \rho}$ and $\Omega_{\alpha}^{\rho \rho}$ are non-negative. Therefore, the relation (87) shows that Pauli blocking and periodic driving are both sources of precision, which reduce the minimal amount of entropy that must be produced to generate an arbitrary matter current $J_{\alpha}^{\rho}$ with given uncertainty $\epsilon_{\alpha}^{\rho}$. These two mechanisms of reducing the thermodynamic cost of precision have been described separately before for different classes of systems; see, for instance, Refs. [47,91,92] and Refs. [48,93-100], respectively. Our uncertainty relation (87) now accounts for both of them in a unified manner through additive corrections.

For heat currents, the situation is slightly different. The corresponding Pauli corrections $\Psi_{\alpha}^{q q}$ are non-negative and therefore universally suppress the lower bound on the product $\sigma\left(\epsilon_{\alpha}^{q}\right)^{2}$ compared to the quasiclassical limit, where $\Psi_{\alpha}^{q q}=0$; this observation is in line with earlier results; see Ref. [101]. The sign of the driving corrections $\Omega_{\alpha}^{q q}$, however, depends on the structure of the Floquet scattering amplitudes of the sample. Periodic driving can thus either reduce or increase the minimal thermodynamic cost of heat transport at given precision.

We note that the bound (82), to which the relation (87) reduces in the quasiclassical limit and without periodic driving, can be asymptotically saturated in systems with infinitely many terminals and strong biases [47]. Hence, for matter currents, also the uncertainty relation (87) and the stronger bound (78a) can be regarded as tight. Whether these bounds are also tight for heat currents is an open question.

\section{Thermodynamic inference}

Although the corrections $\Psi_{\alpha}^{x x}$ and $\Omega_{\alpha}^{x x}$ admit a transparent physical interpretation, it is not clear how they can be measured. Before the generalized uncertainty relation (87) can be used for thermodynamic inference, it is therefore necessary to link these quantities to experimentally accessible observables. For matter currents, such a connection is provided by the bounds,

$$
\Psi_{\alpha}^{\rho \rho} \leq \sigma / 2 \quad \text { and } \quad \Omega_{\alpha}^{\rho \rho} \leq 2 T_{\alpha} / h,
$$

which ultimately follow from the unitarity conditions (8), see Lemmas 4 and 5 of Appendix B for details. Inserting these bounds into Eq. (87) yields

$$
\frac{\sigma\left(P_{\alpha \alpha}^{\rho \rho}+\sigma / 2+2 T_{\alpha} / h\right)}{\left(J_{\alpha}^{\rho}\right)^{2}} \geq \psi^{*} .
$$

This relation provides a powerful tool for thermodynamic inference. Specifically, upon measuring an arbitrary matter current $J_{\alpha}^{\rho}$, its zero-frequency noise $P_{\alpha \alpha}^{\rho \rho}$, and the temperature $T_{\alpha}$ of the corresponding reservoir, one obtains the lower bound,

$$
\sigma \geq \sigma_{\alpha}^{*} \equiv\left|J_{\alpha}^{\rho}\right|\left(\sqrt{\phi_{\alpha}^{2}+2 \psi^{*}}-\left|\phi_{\alpha}\right|\right),
$$

on the dissipation rate $\sigma$, where $\phi_{\alpha} \equiv\left(h P_{\alpha \alpha}^{\rho \rho}+2 T_{\alpha}\right) / h J_{\alpha}^{\rho}$ is a modified Fano factor. This bound holds, within the limits of the scattering approach to quantum transport, for any sample and driving protocols, arbitrary driving frequencies, arbitrary voltage and temperature biases, and in the presence of magnetic fields. It therefore makes it possible to derive universal constraints on otherwise unaccessible figures of merit of coherent transport devices. For instance, upon recalling the setups of Secs. V C and V B, the energy uptake $U$ of a parametric quantum pump and the efficiency of a quantum motor $\eta_{\mathrm{m}}$ can be bounded as

$$
\begin{gathered}
U \geq T \mathcal{T}\left|J^{\rho}\right|\left(\sqrt{\phi^{2}+2 \psi^{*}}-|\phi|\right), \\
\eta_{\mathrm{m}} \leq 1-\frac{T}{\Delta \mu}\left(\sqrt{\phi^{2}+2 \psi^{*}}-|\phi|\right),
\end{gathered}
$$

where the parameter $\phi \equiv\left(h P_{11}^{\rho \rho}+2 T\right) / J^{\rho}$ is experimentally accessible. These relations can be regarded as far-fromequilibrium counterparts of our linear-response bounds (49) and (63).

\section{Quantum generator revisited}

To illustrate our far-from-equilibrium theory, we now return to the quantum generator of Sec. VA. The Floquet scattering amplitudes for this system can be found exactly by solving the corresponding Schrödinger equation; see Appendix D. It is thus straightforward to numerically calculate the quantities,

$$
\begin{aligned}
& Q^{(1)} \equiv \frac{4 \sigma \hat{K}_{1}^{\rho \rho}}{\left(1+\theta_{1}^{\rho}\right)\left(J_{1}^{\rho}\right)^{2}}, \\
& Q^{(2)} \equiv \sigma\left(\epsilon_{1}^{\rho}\right)^{2}\left(1+\Psi_{1}^{\rho \rho} / P_{11}^{\rho \rho}+\Omega_{1}^{\rho \rho} / P_{11}^{\rho \rho}\right),
\end{aligned}
$$



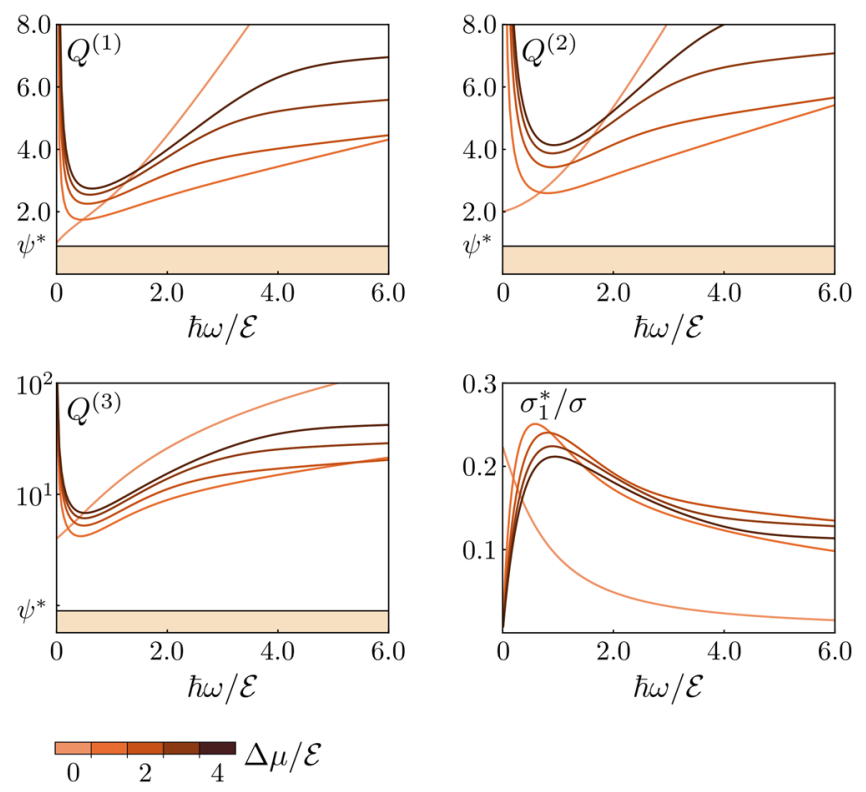

FIG. 5. Quantum generator far from equilibrium. The first three plots from top left to bottom left show the quantities (92a)-(92c) as functions of the driving frequency for different biases. Here, $\mathcal{E} \equiv \hbar^{2} / 2 M l^{2}$ denotes the natural energy scale of the device with $M$ being the carrier mass and $l$ the circumference of the loop, cf. Fig. 2. The shaded areas indicate the bounds (78a), (87), and (89), respectively, for $Q^{(1)}, Q^{(2)}, Q^{(3)}$. The last plot shows the lower bound on entropy production (92d) in units of the actual dissipation rate $\sigma$. For all plots, we have set $\mu=0, \mu_{1} / \mathcal{E}=$ $\Delta \mu / \mathcal{E}=0,1, \ldots, 4$ and $T / \mathcal{E}=1 / 2$.

$$
\begin{aligned}
Q^{(3)} & \equiv \frac{\sigma\left(P_{11}^{\rho \rho}+\sigma / 2+2 T / h\right)}{\left(J_{1}^{\rho}\right)^{2}}, \\
\sigma_{1}^{*} & =\left|J_{1}^{\rho}\right|\left(\sqrt{\phi_{1}^{2}+2 \psi^{*}}-\phi_{1}\right),
\end{aligned}
$$

which are plotted in Fig. 5.

According to the relations (78), (87), and (89), the dimensionless coefficients $Q^{(1)}, Q^{(2)}$, and $Q^{(3)}$ are bounded from below by $\psi^{*}$. The coefficient $Q^{(1)}$ indeed comes close to this bound for small biases and driving frequencies. In fact, we have $Q^{(1)} \rightarrow 1$ for $\Delta \mu, \omega \rightarrow 0$, which confirms our previous observation that the linear-response counterpart (45) of the bound (78) is saturated for the quantum generator. After passing through its minimum, $Q^{(1)}$ grows monotonically with the driving frequency. Hence, the bound (78) becomes less and less tight when the system is driven faster. The coefficients $Q^{(2)}$ and $Q^{(3)}$ show a qualitatively similar dependence on the driving frequency. However, their minimums do not become smaller than 2 and 4 , respectively. Finally, we find that the figure $\sigma_{1}^{*}$, at best, underestimates the actual dissipation rate by about a factor of 4. This result proves that the bound (89) is generally strong enough to predict the correct order of magnitude for $\sigma$.

\section{E. Comparison with earlier results}

Thermodynamic uncertainty relations are currently a subject of active research in stochastic thermodynamics; see Refs. [89,90] for recent reviews. To put our bounds in context with these developments, we compare them with the four relations,

$$
\begin{aligned}
Q_{\mathrm{cl}}^{\rho, \alpha} & \equiv \frac{\sigma P_{\alpha \alpha}^{\rho \rho}}{\psi^{*}\left(J_{\alpha}^{\rho}\right)^{2}} \geq 1, \\
Q_{\omega}^{x, \alpha} & \equiv \frac{\sigma P_{\alpha \alpha}^{x x}}{2\left(J_{\alpha}^{x}-\omega \partial_{\omega} J_{\alpha}^{x}\right)^{2}} \geq 1, \\
Q_{\mathrm{FT}}^{x, \alpha} & \equiv \frac{(\exp [\mathcal{T} \sigma]-1) P_{\alpha \alpha}^{x x}}{2 \mathcal{T}\left(J_{\alpha}^{x}\right)^{2}} \geq 1, \\
Q_{\mathrm{hys}}^{x, \alpha} & \equiv \frac{(\exp [\mathcal{T}(\sigma+\tilde{\sigma}) / 2]-1)\left(P_{\alpha \alpha}^{x x}+\tilde{P}_{\alpha \alpha}^{x x}\right)}{\left(J_{\alpha}^{x}+\tilde{J}_{\alpha}^{x}\right)^{2}} \geq 1,
\end{aligned}
$$

which were derived earlier for different classes of systems [47,98,102-105]. For concreteness, we focus again on the quantum generator of Sec. VA, for which the coefficients $Q_{\mathrm{cl}}^{\rho, 1}, Q_{\omega}^{\rho, 1}, Q_{\mathrm{FT}}^{\rho, 1}$, and $Q_{\mathrm{hys}}^{\rho, 1}$ can be easily calculated. The results of this analysis, which are summarized in Fig. 6, lead to the following conclusions.

First, the bound (93a), which was derived for classical ballistic transport without periodic driving [47], is violated at low frequencies. In fact, the coefficient $Q_{\mathrm{cl}}^{\rho 1}$ goes to zero
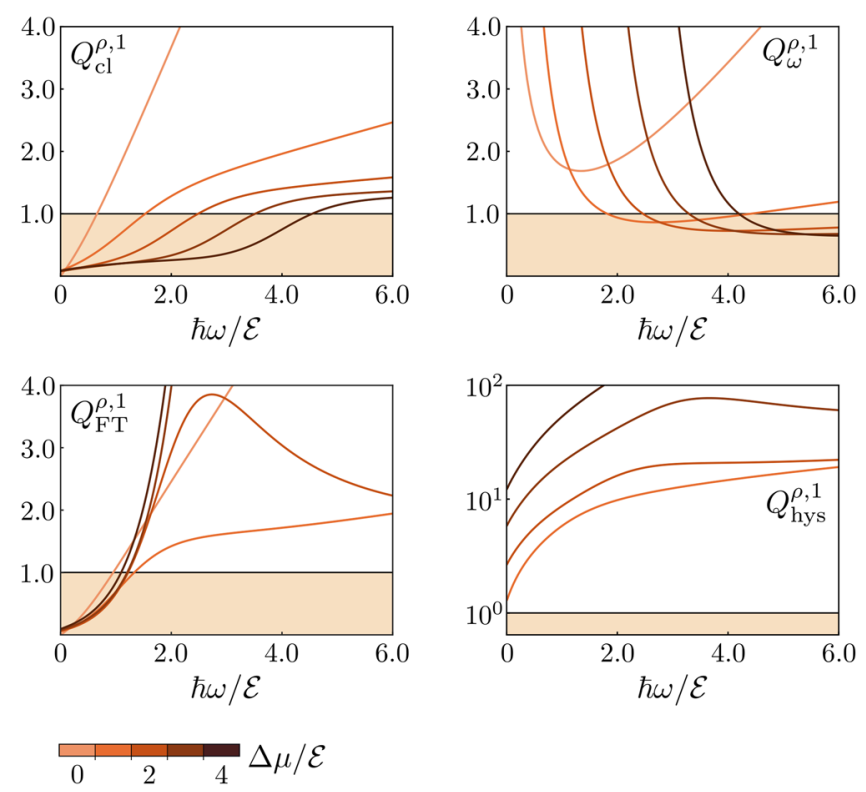

FIG. 6. Quantum generator in perspective. The four plots from top left to bottom right show the coefficients (93a)-(93d) for $x=\rho$ and $\alpha=1$ as functions of the driving frequency for $T / \mathcal{E}=1 / 2, \mu=0$ and different biases $\Delta \mu / \mathcal{E}=0,1, \ldots, 4$, where $\mathcal{E}$ was defined in Fig. 5. In each plot, the shaded area indicates the putative lower bound 1 on the corresponding coefficient. 
for $\Delta \mu, \omega \rightarrow 0$. This behavior can be understood by observing that the driving corrections (86) do, in contrast to the Pauli corrections (85), not vanish in adiabatic equilibrium. Instead, we have

$\left.\Omega_{\alpha}^{x x}\right|_{\mathrm{eq}}=\frac{2}{h} \int_{0}^{\infty} d E\left(\zeta_{E}^{x}\right)^{2}\left(\left\langle\left\langle\left|\mathcal{S}_{E, \mathbf{V}}^{\alpha \alpha}\right|^{2}\right\rangle\right\rangle-\mid\left\langle\left.\left\langle\mathcal{S}_{E, \mathbf{V}}^{\alpha \alpha}\right\rangle\right|^{2}\right) f_{E}^{\prime}\right.$,

and thus $\left.\Omega_{1}^{\rho \rho}\right|_{\text {eq }}=2 T \varphi / h(1+\varphi)>0$, with $\varphi=\exp [\mu / T]$ the quantum generator. We recall that the notation $\left.\cdots\right|_{\text {eq }}$ indicates the limit $F_{\alpha}^{x}, \omega \rightarrow 0$. In general, the corrections $\Omega_{\alpha}^{x x}$ vanish only if the driving fields are switched off, that is, if their amplitudes rather than their frequency go to zero. In this limit, the quantum generator does not produce any current and the bound (93a) becomes trivial.

The second relation (93b) was originally derived for periodically modulated Markov jump processes [98]. In linear response, it holds also for coherent transport, provided that the frozen scattering amplitudes obey the symmetry $\mathcal{S}_{E, \mathbf{V}}^{\alpha \beta}=\mathcal{S}_{E, \mathbf{V}}^{\beta \alpha}$, as is the case for the quantum generator, cf. Eq. (47); for details, see Appendix C. Far from equilibrium, however, the bound (93b) can be violated, as the second plot in Fig. 6 proves.

The fluctuation theorem uncertainty relation (93c) follows from a general symmetry argument. Specifically, it holds if the joint probability distribution of the entropy production per cycle $\mathcal{T} \sigma$ and the accumulated current $\mathcal{T} J_{\alpha}^{x}$ obeys a strong detailed fluctuation theorem [102]. In general, such a relation holds only if no magnetic fields are applied to the system and the driving protocols are symmetric under time reversal. These conditions do not apply to the quantum generator. As a result, the third plot in Fig. 6 shows clear violations of the bound (93c) at low frequencies.

The hysteretic uncertainty relation (93d) provides a generalization of Eq. (93c). The restriction to systems without magnetic fields and symmetric driving protocols is thereby removed by considering the actual thermodynamic process together with its time-reversed counterpart, which is denoted by a tilde [103,104]; in coherent transport, quantities with a tilde are obtained from original ones by replacing $S_{E n, E}^{\alpha \beta}$ with $\mathrm{T}_{\mathbf{B}} \mathrm{T}_{\mathbf{V}} S_{E n, E}^{\alpha \beta}=S_{E, E n}^{\beta \alpha}$. The last plot in Fig. 6 shows that the hysteretic uncertainty relation (93d) is not violated for the quantum generator. Whether or not it holds for coherent transport in general remains an open question at this point. In any case, the relation (93d) has only limited predictive power in practice. First, it is inapplicable if the time-reversed process cannot be realized. Second, since it involves only the symmeterized variable $\sigma+\tilde{\sigma}$, it cannot be used to bound the entropy production $\sigma$ of the actual transport process. Third, if $\tilde{J}_{\alpha}^{x}=-J_{\alpha}^{x}$, as is the case for $J^{\rho}$ in the setup of the quantum generator for $\Delta \mu=0$, Eq. (93d) reduces to the trivial bound $\sigma+\tilde{\sigma} \geq 0$. By contrast, our relation (90) still yields a nontrivial lower bound on $\sigma$ in this situation.
In summary, our case study shows that the new bounds (78), (87), and (89), which fully incorporate quantum effects and do not rely on special symmetries, go beyond the earlier results (93). In particular, Eq. (89) provides a universal bound on entropy production that depends only on parameters that can be measured in a given setup without having to realize the time-reversed transport process.

\section{F. Autonomous systems}

In the previous section, we considered our far-fromequilibrium bounds on coherent transport in the context of earlier results for periodically driven thermodynamic processes. However, our theory also has profound implications for autonomous systems, which can be established as follows. If no time-dependent fields are applied to the sample, the driving correction (86) is zero. The bounds (87) and (88) then imply the new relation:

$$
\frac{\sigma\left(P_{\alpha \alpha}^{\rho \rho}+\sigma / 2\right)}{\left(J_{\alpha}^{\rho}\right)^{2}}=\sigma\left(\epsilon_{\alpha}^{\rho}\right)^{2}+\frac{\sigma^{2}}{2\left(J_{\alpha}^{\rho}\right)^{2}} \geq \psi^{*} .
$$

This result shows that Pauli blocking as a source of precision can be incorporated into the classical uncertainty relation (82), which otherwise can be violated in the quantum regime $[47,91,92]$, through the universal correction $\sigma^{2} / 2\left(J_{\alpha}^{\rho}\right)^{2}$. Quite remarkably, this quantum shift does not depend on any additional parameters. As a result, the relation (95) leads to a bound on entropy production,

$$
\sigma \geq\left|J_{\alpha}^{\rho}\right|\left(\sqrt{\mathrm{F}_{\alpha}^{2}+2 \psi^{*}}-\left|\mathrm{F}_{\alpha}\right|\right)
$$

that involves only the current $J_{\alpha}^{\rho}$ and the standard zerofrequency Fano factor $\mathrm{F}_{\alpha} \equiv P_{\alpha \alpha}^{\rho \rho} / J_{\alpha}^{\rho}$. Like the relation (90), this bound holds for any coherent multiterminal conductor, arbitrary electric and thermal biases, and in presence of external magnetic fields.

Finally, we note that, because the bound (88) on the driving corrections $\Omega_{\alpha}^{\rho \rho}$ is independent of the driving strength, the relations (95) and (96) cannot be recovered from their more general counterparts Eqs. (89) and (90). This observation may indicate that the bounds (89) and (90) can be further optimized. We leave it to future research to probe whether such refined bounds exist. Furthermore, it remains yet an open problem whether operationally accessible bounds on entropy production, similar to the ones given in Eqs. (90) and (96), can be formulated in terms of individual heat currents.

\section{SUMMARY}

Starting from the scattering approach to quantum transport, we have developed a universal thermodynamic framework for coherent conductors that are driven by 
thermochemical gradients and periodically changing electromagnetic fields, whose frequency plays the role of an additional thermodynamic force. Focusing on the linearresponse regime, we have shown that this framework can be equipped with consistent generalizations of the OnsagerCasimir relations and the fluctuation-dissipation theorem. As our first key result, we have derived a family of thermodynamic bounds on matter and heat currents, which go beyond the second law, hold for arbitrary samples and driving protocols, and involve only experimentally accessible quantities.

From a conceptual point of view, these bounds prove that transport without dissipation is impossible in conventional coherent conductors, even in the presence of reversible currents, which generically occur in systems with broken time-reversal symmetry and do not contribute to the average entropy production. From a practical perspective, they provide powerful tools of thermodynamic inference. In particular, they make it possible to determine modelindependent lower bounds on the total dissipation rate, which is generally difficult to access experimentally, by measuring the electric currents in the individual terminals of the conductor.

When applied to mesoscopic devices, our bounds lead to nontrivial relations between key figures of merit, which provide both universal benchmarks for theoretical models and a new avenue to estimate the performance of experimental realizations of nanomachines, whose output or input cannot be measured directly. We have illustrated this method for parametric quantum pumps and adiabatic quantum motors, where in both cases a mechanical quantity was bounded in terms of an easy-to-measure electric current. Beyond these examples, our results are applicable to any system that can be described as a coherent multiterminal conductor, including thermoelectric heat engines and refrigerators, which have gained much attention in recent years [36-49]. Our work thus provides a versatile toolbox for both theoretical and experimental studies seeking to develop powerful and efficient quantum transport devices.

Going beyond the linear-response regime, in the second part of this article, we have derived a family of thermodynamic bounds on matter and heat currents that hold for arbitrary thermochemical gradients and driving frequencies. These relations imply a thermodynamic uncertainty relation for coherent transport, which accounts for quantum effects and periodic driving in a unified manner, and a fully universal bound on entropy production that depends only on experimentally accessible parameters. This bound, which is our second key result, can be directly used for thermodynamic inference far from equilibrium. In fact, it can be determined by measuring a single electric current, its zero-frequency noise, and, for systems with periodic driving, the temperature of the corresponding reservoir. Thus, we are now able to pass the baton to the experimentalists to test our theoretical results and to utilize their wide-ranging applicability to further explore the quantum thermodynamics of coherent transport devices.

\section{ACKNOWLEDGMENTS}

E. P. acknowledges helpful comments from V. Kashcheyevs. K. B. thanks K. Saito for insightful discussions. E. P. acknowledges support from the Vilho, Yrjö and Kalle Foundation of the Finnish Academy of Science and Letters through the grant for doctoral studies. C. F. acknowledges support from Academy of Finland through the Finnish Centre of Excellence in Quantum Technology (Projects No. 312057 and No. 312299) and project No. 308515. M. M. acknowledges the warm hospitality of Aalto University, support from the Aalto Science Institute through its Visiting Fellow Programme, and support from the Ministry of Education and Science of Ukraine (Project No. 0119U002565). K. B. acknowledges support from the Academy of Finland (Contract No. 296073), the Japan Society for the Promotion of Science through a Postdoctoral Fellowship for Research in Japan (Fellowship ID P19026), the University of Nottingham through a Nottingham Research Fellowship, and from UK Research and Innovation through a Future Leaders Fellowship (Grant Reference: MR/S034714/1). Authors from Aalto University are associated with the local Centre for Quantum Engineering.

\section{APPENDIX A: KINETIC COEFFICIENTS}

In this Appendix, we derive the formulas (22) for the linear-response coefficients in the frequency picture, that is, with the driving frequency playing the role of an additional affinity. We then compare this approach with the amplitude picture, where the mechanical affinity corresponds to the strength of the applied periodic fields.

\section{Frequency picture}

We first recall that the thermochemical currents and the photon current are given by

$$
\begin{aligned}
J_{\alpha}^{x} & =\frac{1}{h} \int_{0}^{\infty} d E \sum_{\beta} \sum_{n}\left(\zeta_{E}^{x, \alpha} \delta_{\alpha \beta} \delta_{n 0}-\zeta_{E n}^{x, \alpha}\left|S_{E n, E}^{\alpha \beta}\right|^{2}\right) f_{E}^{\beta} \\
\zeta_{E}^{x, \alpha} & =\zeta_{E}^{x}-\delta_{x q} T F_{\alpha}^{\rho}, \\
\zeta_{E}^{x, \alpha} & =\zeta_{E}^{x}-\delta_{x q} T\left(F_{\alpha}^{\rho}-n F^{\omega}\right), \quad \text { and } \\
J^{\omega} & =\frac{1}{h} \int_{0}^{\infty} d E \sum_{\alpha \beta} \sum_{n} n\left|S_{E n, E}^{\alpha \beta}\right|^{2} f_{E}^{\beta},
\end{aligned}
$$

where $\zeta_{E}^{\rho} \equiv 1, \zeta_{E}^{q} \equiv E-\mu$, and $\zeta^{\omega}=1 / \omega$. Inserting the expansion of the Fermi function (20) and the expansions

$$
\begin{aligned}
& \sum_{n}\left|S_{E n, E}^{\alpha \beta}\right|^{2}=\mathcal{X}_{0}+\frac{T F^{\omega}}{2} \mathcal{X}_{1}, \\
& \sum_{n} n\left|S_{E n, E}^{\alpha \beta}\right|^{2}=\mathcal{Y}_{0}+\frac{T F^{\omega}}{2} \mathcal{Y}_{1},
\end{aligned}
$$


and collecting first-order contributions in the affinities then yields $F_{\alpha}^{x}$ and $F^{\omega}$ yields

$$
\begin{aligned}
L_{\alpha \beta}^{x y} & =\frac{1}{h} \int_{0}^{\infty} d E \zeta_{E}^{x} \zeta_{E}^{y}\left(\delta_{\alpha \beta}-\mathcal{X}_{0}\right) f_{E}^{\prime}, \\
L_{\alpha}^{x \omega} & =-\frac{T}{2 h} \int_{0}^{\infty} d E \sum_{\beta}\left(2 \mathcal{Y}_{0} \delta_{x q}+\zeta_{E}^{x} \mathcal{X}_{1}\right) f_{E}, \\
L_{\beta}^{\omega x} & =\frac{1}{h} \int_{0}^{\infty} d E \zeta_{E}^{x} \sum_{\alpha} \mathcal{Y}_{0} f_{E}^{\prime}, \\
L^{\omega \omega} & =\frac{T}{2 h} \int_{0}^{\infty} d E \sum_{\alpha \beta} \mathcal{Y}_{1} f_{E} .
\end{aligned}
$$

Note that the zeroth-order terms vanish, as can be shown with the help of the unitarity conditions (37) for the frozen scattering amplitudes.

Upon inserting the expressions (B2a)-(B2d) for $\mathcal{X}_{0}, \mathcal{X}_{1}$, $\mathcal{Y}_{0}$, and $\mathcal{Y}_{1}$ in Eqs. (A3), we arrive at

$$
\begin{aligned}
L_{\alpha \beta}^{x y}= & \frac{1}{h} \int_{0}^{\infty} d E \zeta_{E}^{x} \zeta_{E}^{y}\left(\delta_{\alpha \beta}-\left\langle\left\langle\left|\mathcal{S}_{E, \mathbf{V}}^{\alpha \beta}\right|^{2}\right\rangle\right\rangle\right) f_{E}^{\prime}, \\
L_{\alpha}^{\omega x}= & \frac{T}{2 h} \int_{0}^{\infty} d E \sum_{\beta}\left(2 \zeta^{\omega} \operatorname{Im}\left[\left\langle\dot{\mathcal{S}}_{E, \mathbf{V}}^{\alpha \beta} \mathcal{S}_{E, \mathbf{V}}^{\alpha \beta *}\right\rangle\right] \delta_{x q}\right. \\
& \left.-4 \zeta_{E}^{x} \operatorname{Re}\left[\left\langle\left\langle\mathcal{S}_{E, \mathbf{V}}^{\alpha \beta *} \mathcal{A}_{E}^{\alpha \beta}\right\rangle\right\rangle\right]+\zeta_{E}^{x} \zeta^{\omega} \partial_{E} \operatorname{Im}\left[\left\langle\dot{\mathcal{S}}_{E, \mathbf{V}}^{\alpha \beta} \mathcal{S}_{E, \mathbf{V}}^{\alpha \beta *}\right\rangle\right]\right) f_{E} \\
= & \frac{T}{h} \int_{0}^{\infty} d E \sum_{\beta}\left(\zeta^{\omega} \operatorname{Im}\left[\left\langle\dot{\mathcal{S}}_{E, \mathbf{V}}^{\alpha \beta} \mathcal{S}_{E, \mathbf{V}}^{\alpha \beta *}\right\rangle\right] \delta_{x q}\right. \\
& +\zeta_{E}^{x} \zeta^{\omega} \partial_{E} \operatorname{Im}\left[\left\langle\left\langle\dot{\mathcal{S}}_{E, \mathbf{V}}^{\alpha \beta} \mathcal{S}_{E, \mathbf{V}}^{\alpha \beta *}\right\rangle\right]\right) f_{E} \\
= & \frac{1}{h} \int_{0}^{\infty} d E \zeta_{E}^{x} \zeta^{\omega} \sum_{\beta} \operatorname{Im}\left[\left\langle\dot{\mathcal{S}}_{E, \mathbf{V}}^{\alpha \beta} \mathcal{S}_{E, \mathbf{V}}^{\alpha \beta *}\right\rangle\right] f_{E}^{\prime},
\end{aligned}
$$$$
\left.L_{\alpha}^{x \omega}=\frac{1}{h} \int_{0}^{\infty} d E \zeta_{E}^{x} \zeta^{\omega} \sum_{\beta} \operatorname{Im}\left[\left\langle\dot{\mathcal{S}}_{E, \mathbf{V}}^{\beta \alpha *} \mathcal{S}_{E, \mathbf{V}}^{\beta \alpha}\right\rangle\right\rangle\right],
$$$$
L^{\omega \omega}=\frac{T}{2 h} \int_{0}^{\infty} d E \zeta^{\omega} \sum_{\alpha \beta}\left(4 \operatorname{Im}\left[\left\langle\left\langle\dot{\mathcal{S}}_{E, \mathbf{V}}^{\alpha \beta *} \mathcal{A}_{E}^{\alpha \beta}\right\rangle\right\rangle\right]\right.
$$$$
+\zeta^{\omega} \partial_{E}\left\langle\left\langle\left|\dot{\mathcal{S}}_{E, \mathbf{V}}^{\alpha \beta}\right|^{2}\right\rangle\right) f_{E}
$$$$
=\frac{1}{2 h} \int_{0}^{\infty} d E\left(\zeta^{\omega}\right)^{2} \sum_{\alpha \beta}\left\langle\left\langle\left|\dot{\mathcal{S}}_{E, \mathbf{V}}^{\alpha \beta}\right|^{2}\right\rangle\right\rangle f_{E}^{\prime} .
$$

Here, we have used the sum rule,

$$
\begin{aligned}
\left.4 \sum_{\beta} \operatorname{Re}\left[\left\langle\mathcal{S}_{E, \mathbf{V}}^{\alpha \beta *} \mathcal{A}_{E}^{\alpha \beta}\right\rangle\right\rangle\right] & =-i \zeta^{\omega} \sum_{\beta} \partial_{E}\left\langle\left\langle\dot{\mathcal{S}}_{E, \mathbf{V}}^{\alpha \beta *} \mathcal{S}_{E, \mathbf{V}}^{\alpha \beta}\right\rangle\right\rangle \\
& =-\zeta^{\omega} \sum_{\beta} \partial_{E} \operatorname{Im}\left[\left\langle\dot{\mathcal{S}}_{E, \mathbf{V}}^{\alpha \beta} \mathcal{S}_{E, \mathbf{V}}^{\alpha \beta *}\right\rangle\right],
\end{aligned}
$$

which follows from Eq. (B4b), in the first step of Eq. (A4b); in the second step, we have applied an integration by parts with respect to $E$ noting that $\partial_{E} \zeta_{E}^{x}=\delta_{x q}$ and $\partial_{E} f_{E}=-f_{E}^{\prime} / T$. In Eq. (A4d), we have used that

$$
\left.\sum_{\alpha \beta} \operatorname{Im}\left[\left\langle\dot{\mathcal{S}}_{E, \mathbf{V}}^{\alpha \beta *} \mathcal{A}_{E}^{\alpha \beta}\right\rangle\right]\right]=0
$$

as a consequence of the sum rule (B4c) and the fact that the period average is a linear operation; finally, we have integrated the remaining term by parts.

\section{Amplitude picture}

The amplitude picture provides an alternative way of extending the framework of irreversible thermodynamics to periodically driven coherent conductors. This theory, which is complementary to the one discussed in Sec. III, can be developed along the same lines as for stochastic systems; see Refs. [58-61]. We first divide the single-carrier Hamiltonian that describes the dynamics inside the conductor into two contributions,

$$
H_{t} \equiv H_{0}+\Delta U_{t},
$$

where the free Hamiltonian $H_{0}$ is time independent, the dynamical scattering potential $U_{t}=U_{t+\mathcal{T}}$ accounts for the periodic driving, and the parameter $\Delta$ denotes to the amplitude of this perturbation. The Floquet scattering amplitudes can thus be decomposed as

$$
S_{E n, E}^{\alpha \beta}=\delta_{n 0} S_{E}^{\alpha \beta}+\Delta Z_{E n, E}^{\alpha \beta},
$$

with the first contribution describing elastic scattering events and the second one accounting for inelastic events, which are induced by the time-dependent driving. We now introduce the affinity $F^{w} \equiv \Delta / T$ and the work flux,

$$
J^{w} \equiv \Pi_{\mathrm{ac}} / \Delta=\frac{\Delta}{\mathcal{T}} \int_{0}^{\infty} d E \sum_{\alpha \beta} \sum_{n} n\left|Z_{E n, E}^{\alpha \beta}\right|^{2} f_{E}^{\beta},
$$

where the second expression is obtained by inserting Eq. (A8) into Eq. (11). With these definitions, the total rate of entropy production (16) assumes the canonical bilinear form:

$$
\sigma=J^{w} F^{w}+\sum_{\alpha} \sum_{x} J_{\alpha}^{x} F_{\alpha}^{x} .
$$

The frequency and the amplitude picture are equivalent on the general level. Their corresponding linear-response theories, however, describe two physically different regimes, where either the speed or the strength of the periodic driving is small. This difference becomes particularly clear from the kinetic coefficients in the amplitude picture, which are given by [106] 
$\left.G_{\alpha \beta}^{x y} \equiv \partial_{F_{\beta}^{y}} J_{\alpha}^{x}\right|_{\mathrm{eq}}=\frac{1}{h} \int_{0}^{\infty} d E \zeta_{E}^{x} \zeta_{E}^{y}\left(\delta_{\alpha \beta}-\left|S_{E}^{\alpha \beta}\right|^{2}\right) f_{E}^{\prime}$,

$\left.G_{\alpha}^{w x} \equiv \partial_{F^{w}} J_{\alpha}^{x}\right|_{\mathrm{eq}}=0$

$\left.G_{\alpha}^{x w} \equiv \partial_{F_{\alpha}^{x}} J^{w}\right|_{\mathrm{eq}}=0$

$\left.G^{w w} \equiv \partial_{F^{w}} J^{w}\right|_{\text {eq }}=\frac{T}{\mathcal{T}} \int_{0}^{\infty} d E \sum_{\alpha \beta} n\left|\mathcal{Z}_{E n, E}^{\alpha \beta}\right|^{2} f_{E}$,

with $\left.\mathcal{Z}_{E n, E}^{\alpha \beta} \equiv Z_{E n, E}^{\alpha \beta}\right|_{\Delta=0}$. Hence, the thermochemical variables, $F_{\alpha}^{x}$ and $J_{\alpha}^{x}$, decouple from the mechanical ones, $F^{w}$ and $J^{w}$, in the weak-driving regime. By contrast, this coupling persists in slow-driving regime, provided that the driving protocols are not symmetric under time reversal, cf. Sec. III C.

\section{APPENDIX B: SOME USEFUL LEMMAS}

In this Appendix, we collect a series of sum rules for the Floquet scattering amplitudes in the slow-driving regime along with sketches of their proofs. Further details on the derivations may be found in Refs. [7,32].

Lemma 1.-Let $S_{E n, E}^{\alpha \beta}$ be the Floquet scattering amplitudes of a multiterminal conductor that is subject to the periodic driving fields $\mathbf{V}$ with frequency $\omega \equiv 2 \pi / \mathcal{T}$, then

$$
\begin{aligned}
\sum_{n}\left|S_{E n, E}^{\alpha \beta}\right|^{2} & =\mathcal{X}_{0}+\frac{\hbar \omega}{2} \mathcal{X}_{1}, \\
\sum_{n} n\left|S_{E n, E}^{\alpha \beta}\right|^{2} & =\mathcal{Y}_{0}+\frac{\hbar \omega}{2} \mathcal{Y}_{1},
\end{aligned}
$$

up to second-order corrections in $\hbar \omega$, with

$$
\begin{aligned}
& \mathcal{X}_{0}=\left\langle\left\langle\left|\mathcal{S}_{E, \mathbf{V}}^{\alpha \beta}\right|^{2}\right\rangle\right\rangle, \\
& \mathcal{X}_{1}=4 \operatorname{Re}\left[\left\langle\left\langle\mathcal{S}_{E, \mathbf{V}}^{\alpha \beta *} \mathcal{A}_{E}^{\alpha \beta}\right\rangle\right]-\frac{1}{\omega} \partial_{E} \operatorname{Im}\left[\left\langle\left\langle\dot{\mathcal{S}}_{E, \mathbf{V}}^{\alpha \beta} \mathcal{S}_{E, \mathbf{V}}^{\alpha \beta *}\right\rangle\right]\right]\right. \\
& \mathcal{Y}_{0}=-\frac{1}{\omega} \operatorname{Im}\left[\left\langle\left\langle\dot{\mathcal{S}}_{E, \mathbf{V}}^{\alpha \beta} \mathcal{S}_{E, \mathbf{V}}^{\alpha \beta *}\right\rangle\right],\right. \\
& \mathcal{Y}_{1}=\frac{4}{\omega} \operatorname{Im}\left[\left\langle\left\langle\dot{\mathcal{S}}_{E, \mathbf{V}}^{\alpha \beta *} \mathcal{A}_{E}^{\alpha \beta}\right\rangle\right\rangle\right]+\frac{1}{\omega^{2}} \partial_{E}\left\langle\left\langle\left|\dot{\mathcal{S}}_{E, \mathbf{V}}^{\alpha \beta}\right|^{2}\right\rangle\right\rangle .
\end{aligned}
$$

Here, $\mathcal{S}_{E, \mathbf{V}}^{\alpha \beta}$ denotes the frozen scattering amplitudes, $\mathcal{A}_{E}^{\alpha \beta}$ the nonadiabatic corrections defined in Eq. (21), and $\langle\cdots\rangle$ indicates the time average over one period $\mathcal{T}$.

Proof--Insert the low-frequency expansion (21) for $S_{E n, E}^{\alpha \beta}$ and collect all zeroth- and first-order terms in $\hbar \omega$. Perform the sum over all integers $n$ by using the symbolic identity,

$$
\sum_{n} n^{k} e^{i n \omega\left(t-t^{\prime}\right)}=\frac{\mathcal{T}}{(i \omega)^{k}} \partial_{t}^{k} \delta_{t-t^{\prime}}
$$

where $k=0,1, \ldots$ and $t, t^{\prime} \in[0, \mathcal{T})$. Integrate by parts with respect to $t$ as needed.

Lemma 2.- The frozen scattering amplitudes and the nonadiabatic corrections obey the joint sum rules,

$$
\begin{aligned}
& 2 i \omega \sum_{\alpha}\left(\mathcal{S}_{E, \mathbf{V} t}^{\alpha \beta} \mathcal{A}_{E, t}^{\alpha \gamma *}+\mathcal{S}_{E, t}^{\alpha \gamma *} \mathcal{A}_{E, \mathbf{V} t}^{\alpha \beta}\right) \\
&= \sum_{\alpha}\left(\dot{\mathcal{S}}_{E, \mathbf{V} t}^{\alpha \beta} \partial_{E} \mathcal{S}_{E, \mathbf{V} t}^{\alpha \gamma *}-\dot{\mathcal{S}}_{E, \mathbf{V} t}^{\alpha \gamma *} \partial_{E} \mathcal{S}_{E, \mathbf{V} t}^{\alpha \beta}\right), \\
& 2 i \omega \sum_{\alpha}\left(\mathcal{S}_{E, \mathbf{V} t}^{\beta \alpha} \mathcal{A}_{E, t}^{\gamma \alpha *}+\mathcal{S}_{E, \mathbf{V} t}^{\gamma \alpha *} \mathcal{A}_{E, t}^{\beta \alpha}\right) \\
&=\sum_{\alpha}\left(\dot{\mathcal{S}}_{E, \mathbf{V} t}^{\gamma \alpha *} \partial_{E} \mathcal{S}_{E, \mathbf{V} t}^{\beta \alpha}-\dot{\mathcal{S}}_{E, \mathbf{V} t}^{\beta \alpha} \partial_{E} \mathcal{S}_{E, \mathbf{V} t}^{\gamma \alpha *}\right), \\
& \sum_{\alpha \beta} \operatorname{Im}\left[\dot{\mathcal{S}}_{E, \mathbf{V} t}^{\alpha \beta *} \mathcal{A}_{E, t}^{\alpha \beta}\right]=0,
\end{aligned}
$$

for any $t \in[0, \mathcal{T})$.

Proof.-We begin with the sum rule (B4a). Inserting the expansion (21) into the unitarity condition (8a), collecting first-order terms in $\hbar \omega$, and carrying out the sum over $n$ using Eq. (B3) shows that

$$
\begin{aligned}
& \int_{0}^{\mathcal{T}} d t\left\{\sum _ { \alpha } \left(2 \dot{\mathcal{S}}_{E, \mathbf{V} t}^{\alpha \beta} \partial_{E} \mathcal{S}_{E, \mathbf{V} t}^{\alpha \gamma *}+\mathcal{S}_{E, \mathbf{V} t}^{\alpha \beta} \partial_{E} \dot{\mathcal{S}}_{E, \mathbf{V} t}^{\alpha \gamma *}+\mathcal{S}_{E, \mathbf{V} t}^{\alpha \gamma *} \partial_{E} \dot{\mathcal{S}}_{E, \mathbf{V} t}^{\alpha \beta}\right.\right. \\
& \left.\left.-2 i \omega\left(\mathcal{S}_{E, \mathbf{V} t}^{\alpha \beta} \mathcal{A}_{E, t}^{\alpha \gamma *}+\mathcal{S}_{E, \mathbf{V} t}^{\alpha \gamma *} \mathcal{A}_{E, t}^{\alpha \beta}\right)\right)\right\} e^{i m \omega t}=0,
\end{aligned}
$$

for every integer $m$ and any indices $\beta$ and $\gamma$. This condition can be met only if the expression inside the curly brackets vanishes for every $t \in[0, \mathcal{T})$, that is, if

$$
2 i \omega\left(\mathbb{A}^{\dagger} \mathbb{S}+\mathbb{A} \mathbb{S}^{\dagger}\right)=2 \mathbb{S}^{\prime \dagger} \dot{\mathbb{S}}+\dot{\mathbb{S}}^{\prime \dagger} \mathbb{S}+\mathbb{S}^{\dagger} \dot{\mathbb{S}}^{\prime}
$$

where we introduced the matrices $\mathbb{S}$ and $\mathbb{A}$ with elements $(\mathbb{S})_{\alpha \beta} \equiv \mathcal{S}_{E, \mathbf{V} t}^{\alpha \beta}$ and $(\mathbb{A})_{\alpha \beta} \equiv \mathcal{A}_{E, t}^{\alpha \beta}$ to simplify the notation and primes indicate derivatives with respect to $E$. Next, we note that the matrix $\mathbb{S}$ is unitary, i.e., $\mathbb{S}^{\dagger} \mathbb{S}=\mathbb{S}^{\dagger}=\mathbb{1}$, owing to the conditions (37). Therefore, we have $\partial_{t} \partial_{E} \mathbb{S}^{\dagger} \mathbb{S}=0$, and writing out the derivatives gives

$$
\dot{\mathbb{S}}^{\prime \dagger} \mathbb{S}+\mathbb{S}^{\dagger} \dot{\mathbb{S}}^{\prime}=-\mathbb{S}^{\prime \dagger} \dot{\mathbb{S}}-\dot{\mathbb{S}}^{\dagger} \mathbb{S}^{\prime}
$$

Inserting this relation into Eq. (B6) yields the result

$$
2 i \omega\left(\mathbb{A}^{\dagger} \mathbb{S}+\mathbb{A}^{\dagger}\right)=\mathbb{S}^{\prime \dagger} \dot{S}-\dot{\mathbb{S}}^{\dagger} \mathbb{S}^{\prime}
$$

which is equivalent to the first sum rule (B4a). The second sum rule (B4b) can be derived along the same lines starting with the unitarity condition (8b). 
To derive the third sum rule (B4c), we first note that

$$
\begin{aligned}
2 i \sum_{\alpha \beta} \operatorname{Im}\left[\dot{\mathcal{S}}_{E, \mathbf{V} t}^{\alpha \beta *} \mathcal{A}_{E, t}^{\alpha \beta}\right] & =\operatorname{tr}\left\{\dot{\mathbb{S}}^{\dagger} \mathbb{A}-\mathbb{A}^{\dagger} \dot{\mathbb{S}}\right\} \\
& =\operatorname{tr}\left\{\mathbb{A} \dot{\mathbb{S}}^{\dagger} \mathbb{S} \mathbb{S}^{\dagger}-\mathbb{A}^{\dagger} \dot{\mathbb{S}} \mathbb{S}^{\dagger} \mathbb{S}\right\} \\
& =\operatorname{tr}\left\{\left(\mathbb{S}^{\dagger} \mathbb{A}+\mathbb{A}^{\dagger} \mathbb{S}\right) \dot{\mathbb{S}}^{\dagger} \mathbb{S}\right\}
\end{aligned}
$$

where we have used that $\dot{\mathbb{S}} \mathbb{S}^{\dagger}=-\mathbb{S} \dot{\mathbb{S}}^{\dagger}$, since $\mathbb{S}$ is unitary. Second, eliminating the matrix $\mathbb{A}$ with the help of the relation (B8) gives

$$
\begin{aligned}
-4 \omega \sum_{\alpha \beta} \operatorname{Im}\left[\dot{\mathcal{S}}_{E, \mathbf{V} t}^{\alpha \beta *} \mathcal{A}_{E, t}^{\alpha \beta}\right] & =\operatorname{tr}\left\{\left(\mathbb{S}^{\prime \dagger} \dot{\mathbb{S}}-\dot{\mathbb{S}}^{\dagger} \mathbb{S}^{\prime}\right) \dot{\mathbb{S}}^{\dagger} \mathbb{S}\right\} \\
& =\operatorname{tr}\left\{\left(\mathbb{S}^{\dagger} \mathbb{S}^{\prime} \dot{\mathbb{S}}^{\dagger} \mathbb{S}-\dot{\mathbb{S}}^{\dagger} \mathbb{S}^{\prime}\right) \dot{\mathbb{S}}^{\dagger} \mathbb{S}\right\} \\
& =0
\end{aligned}
$$

where the second line follows by noting that $\mathbb{S} \mathbb{S}^{\prime \dagger}=-\mathbb{S}^{\prime} \mathbb{S}^{\dagger}$ and $\dot{\mathbb{S}} \mathbb{S}^{\dagger}=-\mathbb{S}^{\dagger}$.

Lemma 3.-The frozen scattering amplitudes can be chosen such that they obey the sum rule,

$$
\sum_{\alpha \beta}\left\langle\left\langle\dot{\mathcal{S}}_{E, \mathbf{V}}^{\alpha \beta} \mathcal{S}_{E, \mathbf{V}}^{\alpha \beta *}\right\rangle\right\rangle=0
$$

Proof.-The frozen scattering matrix admits the spectral decomposition $\mathbb{S}=\sum_{k}\left|\phi_{k}\right\rangle\left\langle\phi_{k}\right| e^{i \phi_{k}}$, where the $\phi_{k}$ are real and the $\left|\phi_{k}\right\rangle$ are normalized orthogonal eigenvectors. Since $\mathbb{S}$ is a $\mathcal{T}$-periodic function of time, the phases $\phi_{k}$ further have to obey the condition $\left\langle\left\langle\dot{\phi}_{k}\right\rangle\right\rangle=\omega M_{k}$ with $M_{k}$ being an integer. It follows that

$$
\begin{aligned}
\sum_{\alpha \beta}\left\langle\left\langle\dot{\mathcal{S}}_{E, \mathbf{V}}^{\alpha \beta} \mathcal{S}_{E, \mathbf{V}}^{\alpha \beta *}\right\rangle\right. & =\left\langle\left\langle\operatorname{tr}\left\{\dot{\mathbb{S}} \mathbb{S}^{\dagger}\right\}\right\rangle\right\rangle \\
& =\sum_{k}\left\langle\left\langle i \dot{\phi}_{k}+\partial_{t}\left\langle\phi_{k} \mid \phi_{k}\right\rangle\right\rangle\right\rangle \\
& =i \omega \sum_{k} M_{k} \equiv i \omega M .
\end{aligned}
$$

Thus, the sum rules (B11) can be enforced by applying the gauge transformation $\mathcal{S}_{E, \mathbf{V}}^{\alpha \beta} \rightarrow \mathcal{S}_{E, \mathbf{V}}^{\alpha \beta} e^{-i M \omega t}$ to the frozen scattering amplitudes.

Lemma 4.- Let $S_{E n, E}^{\alpha \beta}$ be a set of Floquet scattering amplitudes that obey the unitarity conditions (8) and $f_{E}^{\alpha}$ the Fermi function (5), then

$$
\begin{aligned}
\sigma & \equiv \frac{1}{h} \int_{0}^{\infty} d E \sum_{\alpha \beta} \sum_{n}\left(\frac{E_{n}-\mu_{\alpha}}{T_{\alpha}}-\frac{E-\mu_{\beta}}{T_{\beta}}\right)\left|S_{E n, E}^{\alpha \beta}\right|^{2} f_{E}^{\beta} \\
& \geq \frac{2}{h} \int_{0}^{\infty} d E \sum_{\alpha \beta} \sum_{n}\left|S_{E n, E}^{\alpha \beta}\right|^{2}\left(f_{E n}^{\alpha}-f_{E}^{\beta}\right)^{2} .
\end{aligned}
$$

Proof.-We first observe that, using the conditions (8), $\sigma$ can be expressed as [8]

$$
\sigma=\frac{1}{h} \int_{0}^{\infty} d E \sum_{\alpha \beta} \sum_{n}\left|S_{E n, E}^{\alpha \beta}\right|^{2} H_{E n, E}^{\alpha \beta},
$$

with

$$
H_{E n, E}^{\alpha \beta} \equiv \eta\left[f_{E n}^{\alpha}\right]-\eta\left[f_{E}^{\beta}\right]-\eta^{\prime}\left[f_{E n}^{\alpha}\right]\left(f_{E n}^{\alpha}-f_{E}^{\beta}\right),
$$

$\eta[x] \equiv-x \ln [x]-(1-x) \ln [1-x]$, and primes indicating derivatives. Next, we note that, by Taylor's theorem, there exists a $g$ between $f_{E n}^{\alpha}$ and $f_{E}^{\beta}$, such that

$$
H_{E n, E}^{\alpha \beta}=-\frac{\eta^{\prime \prime}[g]}{2}\left(f_{E n}^{\alpha}-f_{E}^{\beta}\right)^{2} .
$$

Since the Fermi function (5) takes only values between 0 and 1 , it follows that $g \in[0,1]$. Therefore, we have $-\eta^{\prime \prime}[g]=1 / g+1 /(1-g) \geq 4$ and $H_{E n, E}^{\alpha \beta} \geq 2\left(f_{E n}^{\alpha}-f_{E}^{\beta}\right)^{2}$. Inserting this bound into Eq. (B14) completes the proof.

Lemma 5.-Let $S_{E n, E}^{\alpha \beta}$ be a set of Floquet scattering amplitudes that obey the unitarity conditions (8) and $f_{E}^{\alpha}$ the Fermi function (5). Then, for $\hat{S}_{E n, E}^{\alpha \beta} \equiv\left(1-\delta_{n 0} \delta_{\alpha \beta}\right) S_{E n, E}^{\alpha \beta}$ and $f_{E}^{\prime \alpha} \equiv f_{E}^{\alpha}\left(1-f_{E}^{\alpha}\right)$, we have

$\Psi_{\alpha}^{\rho \rho} \equiv \frac{1}{h} \int_{0}^{\infty} d E \sum_{\beta} \sum_{n}\left|\hat{S}_{E n, E}^{\alpha \beta}\right|^{2}\left(f_{E n}^{\alpha}-f_{E}^{\beta}\right)^{2} \leq \frac{\sigma}{2}$

and

$$
\Omega_{\alpha}^{\rho \rho} \equiv \frac{2}{h} \int_{0}^{\infty} d E \sum_{n}\left|\hat{S}_{E n, E}^{\alpha \alpha}\right|^{2} f_{E}^{\prime \alpha} \leq \frac{2 T_{\alpha}}{h}
$$

where $\sigma$ is defined in Eq. (B13).

Proof.-For Eq. (B17), observe that

$$
\begin{aligned}
2 \Psi_{\alpha}^{\rho \rho} & \leq \frac{2}{h} \int_{0}^{\infty} d E \sum_{\alpha \beta} \sum_{n}\left|\hat{S}_{E n, E}^{\alpha \beta}\right|^{2}\left(f_{E n}^{\alpha}-f_{E}^{\beta}\right)^{2} \\
& =\frac{2}{h} \int_{0}^{\infty} d E \sum_{\alpha \beta} \sum_{n}\left|S_{E n, E}^{\alpha \beta}\right|^{2}\left(f_{E n}^{\alpha}-f_{E}^{\beta}\right)^{2}
\end{aligned}
$$

and use Lemma 4. For Eq. (B18), note that $f_{E}^{\prime \alpha} \geq 0$ and, by the unitarity conditions (8), $\sum_{n}\left|\hat{S}_{E n, E}^{\alpha \alpha}\right|^{2} \leq$ $\sum_{\beta} \sum_{n}\left|S_{E n, E}^{\beta \alpha}\right|^{2}=1$, such that

$$
\Omega_{\alpha}^{\rho \rho} \leq \frac{2}{h} \int_{0}^{\infty} d E f_{E}^{\prime}=\frac{2 T_{\alpha}}{h} \frac{\varphi_{\alpha}}{1+\varphi_{\alpha}} \leq \frac{2 T_{\alpha}}{h},
$$

where $\varphi_{\alpha} \equiv \exp \left[\mu_{\alpha} / T_{\alpha}\right]$. 


\section{APPENDIX C: THERMODYNAMIC UNCERTAINTY RELATION IN LINEAR RESPONSE}

In this Appendix, we show that the thermodynamic uncertainty relation,

$$
\frac{\sigma P_{\alpha \alpha}^{x x}}{\left(J_{\alpha}^{x}-\omega \partial_{\omega} J_{\alpha}^{x}\right)^{2}} \geq 2
$$

which was derived in Ref. [98] for periodically driven Markov jump processes, holds for coherent transport in linear response if the frozen scattering amplitudes obey the symmetry

$$
\mathcal{S}_{E, \mathbf{V}}^{\alpha \beta}=\mathcal{S}_{E, \mathbf{V}}^{\beta \alpha}
$$

We first recall Sec. III B and note that the symmetry (C2) implies $L_{\alpha}^{x \omega}=-L_{\alpha}^{\omega x}$. As a result, the total rate of entropy production (18) can be divided into two contributions, $\sigma=\sigma_{\mathrm{th}}+\sigma_{\mathrm{ac}}$, with

$$
\begin{aligned}
\sigma_{\mathrm{th}} & \equiv \sum_{\alpha \beta} \sum_{x y} L_{\alpha \beta}^{x y} F_{\alpha}^{x} F_{\beta}^{y} \\
& =\frac{1}{2 h} \int_{0}^{\infty} d E \sum_{\alpha \beta}\left\langle\left\langle\left|\hat{\mathcal{S}}_{E, \mathbf{V}}^{\alpha \beta}\right|^{2}\right\rangle\right\rangle\left(Y_{E}^{\alpha \beta}\right)^{2} f_{E}^{\prime} \geq 0, \\
\sigma_{\mathrm{ac}} & \equiv L^{\omega \omega}\left(F^{\omega}\right)^{2} \geq 0,
\end{aligned}
$$

where $\hat{\mathcal{S}}_{E, \mathbf{V}}^{\alpha \beta} \equiv\left(1-\delta_{\alpha \beta}\right) \mathcal{S}_{E, \mathbf{V}}^{\alpha \beta}, Y_{E}^{\alpha \beta} \equiv \sum_{x} \zeta_{E}^{x}\left(F_{\alpha}^{x}-F_{\beta}^{x}\right)$, and the second line in Eq. (C3a) follows by using the unitarity conditions (37). We now consider the quadratic form,

$$
\begin{aligned}
\Xi_{\alpha}^{x} \equiv & \sigma_{\text {th }}+2 G \sum_{\beta} \sum_{y} L_{\alpha \beta}^{x y} F_{\beta}^{y}+G^{2} L_{\alpha \alpha}^{x x} \\
= & \frac{1}{2 h} \int_{0}^{\infty} d E \sum_{\beta \neq \alpha}\left\{\sum_{\gamma \neq \alpha}\left\langle\left\langle\left|\hat{\mathcal{S}}_{E, \mathbf{V}}^{\beta \gamma}\right|^{2}\right\rangle\right\rangle\left(Y_{E}^{\alpha \beta}\right)^{2}\right. \\
& \left.+2\left\langle\left\langle\left|\hat{\mathcal{S}}_{E, \mathbf{V}}^{\alpha \beta}\right|^{2}\right\rangle\right\rangle\left(\zeta_{E}^{x} G+Y_{E}^{\alpha \beta}\right)^{2}\right\} f_{E}^{\prime},
\end{aligned}
$$

where $G$ is real and otherwise arbitrary. The second expression in Eq. (C4), which follows from the unitarity conditions (37) and the symmetry (C2), proves that $\Xi_{\alpha}^{x}$ is positive semidefinite. Next, recalling the kinetic equations (24) and the fluctuation-dissipation theorem (34), yields

$$
\sum_{\beta} \sum_{y} L_{\alpha \beta}^{x y} F_{\beta}^{y}=J_{\alpha}^{x}-\omega \partial_{\omega} J_{\alpha}^{x}, \quad L_{\alpha \alpha}^{x x}=\left.P_{\alpha \alpha}^{x x}\right|_{\mathrm{eq}} / 2 .
$$

Inserting these identities into the definition of $\Xi_{\alpha}^{x}$ and noting that $\sigma_{\text {th }} \leq \sigma$ since $\sigma_{\text {ac }} \geq 0$ shows that

$$
\sigma+2 G\left(J_{\alpha}^{x}-\omega \partial_{\omega} J_{\alpha}^{x}\right)+\left.G^{2} P_{\alpha \alpha}^{x x}\right|_{\mathrm{eq}} / 2 \geq \Xi_{\alpha}^{x} \geq 0
$$

for any $G$. Minimizing the left-hand side of this inequality with respect to $G$ finally gives the thermodynamic uncertainty relation $(\mathrm{C} 1)$.

\section{APPENDIX D: QUANTUM GENERATOR}

In this Appendix, we derive the exact Floquet scattering amplitudes for the quantum generator discussed in Secs. VA and VI D by solving the corresponding FloquetSchrödinger equation in position representation [8]. We further provide explicit formulas for the quantities entering the relations (93) and (92).

\section{Scattering amplitudes}

The system is parametrized according to Fig. 7. An incoming carrier with energy $E>0$ in the terminal $\alpha$ is described by the scattering state $\left|\phi_{E, t}^{\alpha}\right\rangle$. On the leads, the wave function of this state is given by

$$
\begin{aligned}
& \phi_{E, t}^{\alpha}\left[r_{1}\right]=\delta_{\alpha 1} w_{E}^{-}\left[r_{1}\right]+\sum_{n} C_{E n, E}^{1 \alpha} w_{E n}^{+}\left[r_{1}\right] e^{-i n \omega t}, \\
& \phi_{E, t}^{\alpha}\left[r_{2}\right]=\delta_{\alpha 2} w_{E}^{-}\left[r_{2}\right]+\sum_{n} C_{E n, E}^{2 \alpha} w_{E n}^{+}\left[r_{2}\right] e^{-i n \omega t},
\end{aligned}
$$

where the summations run over all integers, the $C_{E n, E}^{\alpha \beta}$ are yet undetermined complex coefficients, and

$$
w_{E}^{ \pm}[r] \equiv w_{E} \exp \left[ \pm i k_{E} r\right]
$$

denotes a normalized incoming $(-)$ or outgoing $(+)$ plane wave with $w_{E} \equiv \sqrt{M / 2 \pi k_{E} \hbar^{2}}$ and $k_{E} \equiv \sqrt{2 M E / \hbar^{2}}$ [8]; we recall that $M$ denotes the carrier mass. On the loop, the scattering wave function obeys the Floquet-Schrödinger equation,
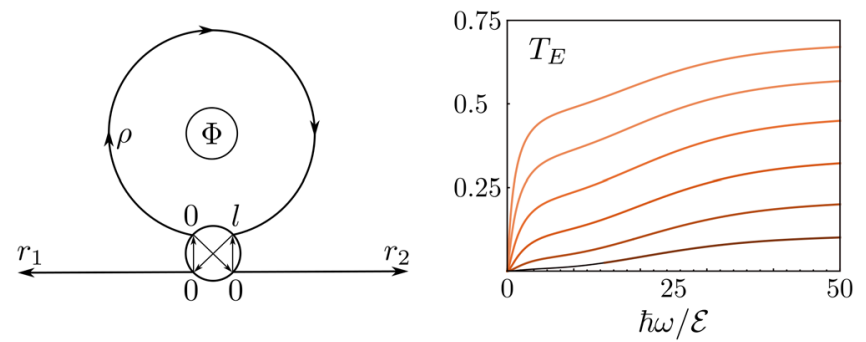

FIG. 7. Scattering theory of a quantum generator. The system consists of two leads, an ideal beam splitter, and a loop with length $l$, which is subject to the magnetic flux $\Phi$. The leads and the loop are parametrized in terms of the coordinates $r_{1}, r_{2} \in$ $[0, \infty)$ and $\rho \in[0, l]$. Incoming waves on the leads 1 and 2 enter the loop at the positions $\rho=0$ and $\rho=l$, respectively; clockwise and counterclockwise propagating waves on the loop exit at the positions $\rho=l$ and $\rho=0$ and become outgoing waves on the leads 1 and 2, respectively. The plot shows how the transmission function (D16b) depends on the driving frequency $\omega$, i.e., the rate at which the magnetic flux $\Phi$ changes, for $E / \mathcal{E}=$ $2^{0} / 10, \ldots, 2^{5} / 10$ from top to bottom. 
$E \phi_{E, t}^{\alpha}[\rho]=-\frac{\hbar^{2}}{2 M}\left(\partial_{\rho}-i \omega t / l\right)^{2} \phi_{E, t}^{\alpha}[\rho]-i \hbar \partial_{t} \phi_{E, t}^{\alpha}[\rho]$,

with respect to the boundary conditions,

$$
\begin{aligned}
\left.\phi_{E, t}^{1}[\rho]\right|_{\rho=0} & =w_{E}+\sum_{n} C_{E n, E}^{21} w_{E n} e^{-i n \omega t}, \\
\left.\phi_{E, t}^{1}[\rho]\right|_{\rho=l} & =\sum_{n} C_{E n, E}^{11} w_{E n} e^{-i n \omega t}, \\
\left.\hat{P}_{t} \phi_{E, t}^{1}[\rho]\right|_{\rho=0} & =w_{E}^{\prime}-\sum_{n} C_{E n, E}^{21} w_{E n}^{\prime} e^{-i n \omega t}, \\
\left.\hat{P}_{t} \phi_{E, t}^{1}[\rho]\right|_{\rho=l} & =\sum_{n} C_{E n, E}^{11} w_{E n}^{\prime} e^{-i n \omega t},
\end{aligned}
$$

and

$$
\begin{aligned}
\left.\phi_{E, t}^{2}[\rho]\right|_{\rho=0} & =\sum_{n} C_{E n, E}^{22} w_{E n} e^{-i n \omega t}, \\
\left.\phi_{E, t}^{2}[\rho]\right|_{\rho=l} & =w_{E}+\sum_{n} C_{E n, E}^{12} w_{E n} e^{-i n \omega t}, \\
\left.\hat{P}_{t} \phi_{E, t}^{2}[\rho]\right|_{\rho=0} & =-\sum_{n} C_{E n, E}^{22} w_{E n}^{\prime} e^{-i n \omega t}, \\
\left.\hat{P}_{t} \phi_{E, t}^{2}[\rho]\right|_{\rho=l} & =-w_{E}^{\prime}+\sum_{n} C_{E n, E}^{12} w_{E n}^{\prime} e^{-i n \omega t},
\end{aligned}
$$

where $w_{E}^{\prime} \equiv w_{E} k_{E}$ and $\hat{P}_{t} \equiv-i \partial_{\rho}-\omega t / l$. Here, we assume that the magnetic flux $\Phi$ increases linearly in time; that is, $\Phi_{t}=\hbar \omega c t / e$, where $c$ denotes the speed of light and $e$ the carrier charge. The parameter $E$ corresponds to the Floquet energy on the loop. The boundary conditions (D4) and (D5) are determined by the beam splitter that connects the loop with the leads [107].

A general solution of Eq. (D3) that is compatible with the boundary conditions (D4) and (D5) is given by

$$
\phi_{E, t}^{\alpha}[\rho]=\sum_{n}\left(a_{n}^{\alpha} \operatorname{Ai}_{E n}[\rho]+b_{n}^{\alpha} \operatorname{Bi}_{E n}[\rho]\right) e^{i(\rho / l-n) \omega t},
$$

where $a_{n}^{\alpha}$ and $b_{n}^{\alpha}$ are arbitrary complex coefficients. The modified Airy functions are thereby defined as

$$
\mathrm{Xi}_{E}[\rho] \equiv \mathrm{Xi}\left[(\hbar \omega / \mathcal{E})^{1 / 3}(\rho / l-E / \hbar \omega)\right]
$$

in terms of the standard Airy functions $\mathrm{Xi} \equiv \mathrm{Ai}, \mathrm{Bi}$ [108]. The parameter $\mathcal{E} \equiv \hbar^{2} / 2 M l^{2}$ sets the natural energy scale of the system. Inserting the solution (D6) into the boundary conditions (D4) and (D5) and collecting Fourier components yields two sets of linear equations, which can be written compactly as

$$
\mathbb{A}_{E n} \mathbf{a}_{n}^{1}=\delta_{n 0} \mathbf{1}+C_{E n, E}^{21} \hat{\mathbf{1}},
$$

$$
\mathbb{A}_{E n-1} \mathbf{a}_{n}^{1}=C_{E n-1, E}^{11} \mathbf{1},
$$

and

$$
\begin{aligned}
\mathbb{A}_{E n} \mathbf{a}_{n}^{2} & =C_{E n, E}^{22} \hat{\mathbf{1}}, \\
\mathbb{A}_{E n-1} \mathbf{a}_{n}^{2} & =\delta_{n 1} \hat{\mathbf{1}}+C_{E n-1, E}^{12} \mathbf{1} .
\end{aligned}
$$

Here, we have used the relation $\mathrm{Xi}_{E n}[l]=\mathrm{Xi}_{E n-1}[0]$. Furthermore, we have introduced the vectors $\mathbf{a}_{n}^{\alpha} \equiv\left(a_{n}^{\alpha}, b_{n}^{\alpha}\right)^{\mathrm{t}}$, $\mathbf{1} \equiv(1,1)^{\mathrm{t}}, \hat{\mathbf{1}} \equiv(1,-1)^{\mathrm{t}}$ and the matrix

$$
\mathbb{A}_{E} \equiv \frac{1}{w_{E}}\left(\begin{array}{cc}
\mathrm{Ai}_{E}[0] & \mathrm{Bi}_{E}[0] \\
-i \mathrm{Ai}_{E}^{\prime}[0] & -i \mathrm{Bi}_{E}^{\prime}[0]
\end{array}\right)
$$

where

$$
\begin{aligned}
\mathrm{Xi}_{E}^{\prime}[\rho] & \equiv \partial_{\rho} \mathrm{Xi}_{E}[\rho] / k_{E} \\
& =(\hbar \omega / \mathcal{E})^{1 / 3}(\mathcal{E} / E)^{1 / 2} \mathrm{Xi}^{\prime}\left[(\hbar \omega / \mathcal{E})^{1 / 3}(\rho / l-E / \hbar \omega)\right],
\end{aligned}
$$

with $\mathrm{Xi}^{\prime} \equiv \mathrm{Ai}^{\prime}, \mathrm{Bi}^{\prime}$ denoting the derivatives of the standard Airy functions [108].

Solving the linear systems (D8) and (D9) yields

$$
\begin{aligned}
& C_{E n, E}^{11}=\delta_{n(-1)} \frac{2}{\mathbf{1}^{\mathrm{t}} \mathbb{A}_{E} \mathbb{A}_{E-1}^{-1} \mathbf{1}}, \\
& C_{E n, E}^{21}=\delta_{n 0} \frac{\hat{\mathbf{1}}^{\mathrm{t}} \mathbb{A}_{E} \mathbb{A}_{E-1}^{-1} \mathbf{1}}{\mathbf{1}^{\mathrm{t}} \mathbb{A}_{E} \mathbb{A}_{E-1}^{-1} \mathbf{1}}, \\
& C_{E n, E}^{22}=\delta_{n 1} \frac{2}{\hat{\mathbf{1}}^{\mathrm{t}} \mathbb{A}_{E} \mathbb{A}_{E 1}^{-1} \hat{\mathbf{1}}}, \\
& C_{E n, E}^{12}=\delta_{n 0} \frac{\mathbf{1}^{\mathrm{t}} \mathbb{A}_{E} \mathbb{A}_{E 1}^{-1} \hat{\mathbf{1}}}{\hat{\mathbf{1}}^{\mathrm{t}} \mathbb{A}_{E} \mathbb{A}_{E \mathbf{1}}^{-1} \hat{\mathbf{1}}} .
\end{aligned}
$$

The Floquet scattering amplitudes can now be determined by inserting these expressions into the ansatz (D1) and comparing the result with the asymptotic boundary conditions for the Floquet scattering wave functions [8], which are given by

$$
\left.\phi_{E, t}^{\alpha}\left[r_{1}\right]\right|_{r_{1} \rightarrow \infty}=\delta_{\alpha 1} w_{E}^{-}\left[r_{1}\right]+\sum_{n} S_{E n, E}^{1 \alpha} w_{E n}^{+}\left[r_{1}\right] e^{-i n \omega t},
$$

$\left.\phi_{E, t}^{\alpha}\left[r_{2}\right]\right|_{r_{2} \rightarrow \infty}=\delta_{\alpha 2} w_{E}^{-}\left[r_{2}\right]+\sum_{n} S_{E n, E}^{2 \alpha} w_{E n}^{+}\left[r_{2}\right] e^{-i n \omega t}$.

Upon observing that the outgoing plane waves become decaying exponentials for negative energies, that is, $w_{E}^{+}[r] \propto \exp \left[-k_{|E|} r\right]$ for $E \leq 0$, we thus arrive at 


$$
\begin{array}{lll}
S_{E n, E}^{11}=0, & S_{E n, E}^{21}=C_{E n, E}^{21} & (E \leq \hbar \omega), \\
S_{E n, E}^{11}=C_{E n, E}^{11}, & S_{E n, E}^{21}=C_{E n, E}^{21} & (E>\hbar \omega), \\
S_{E n, E}^{22}=C_{E n, E}^{22}, & S_{E n, E}^{12}=C_{E n, E}^{12} & (E>0) .
\end{array}
$$

This result leads to the compact expressions,

$$
\begin{aligned}
& \left|S_{E n, E}^{11}\right|^{2}=\delta_{n(-1)} \Theta_{E-1} R_{E-1}, \\
& \left|S_{E n, E}^{21}\right|^{2}=\delta_{n 0}+\delta_{n 0} \Theta_{E-1}\left(T_{E-1}-1\right), \\
& \left|S_{E n, E}^{22}\right|^{2}=\delta_{n 1} R_{E}, \\
& \left|S_{E n, E}^{12}\right|^{2}=\delta_{n 0} T_{E},
\end{aligned}
$$

for the reflection and transmission probabilities, which were used in Sec. VI D. Here, $\Theta$ denotes the Heaviside step function, and the reflection and transmission functions are given by

$$
\begin{aligned}
& R_{E} \equiv \frac{4}{\left|\mathbf{1}^{\mathrm{t}} \mathbb{A}_{E 1} \mathbb{A}_{E}^{-1} \mathbf{1}\right|^{2}}=\frac{4}{\left|\hat{\mathbf{1}}^{\mathrm{t}} \mathbb{A}_{E} \mathbb{A}_{E 1}^{-1} \hat{\mathbf{1}}\right|^{2}}, \\
& T_{E} \equiv \frac{\left|\hat{\mathbf{1}}^{\mathrm{t}} \mathbb{A}_{E 1} \mathbb{A}_{E}^{-1} \mathbf{1}\right|^{2}}{\left|\mathbf{1}^{\mathrm{t}} \mathbb{A}_{E 1} \mathbb{A}_{E}^{-1} \mathbf{1}\right|^{2}}=\frac{\left|\mathbf{1}^{\mathrm{t}} \mathbb{A}_{E} \mathbb{A}_{E 1}^{-1} \hat{\mathbf{1}}\right|^{2}}{\left|\hat{\mathbf{1}}^{\mathrm{t}} \mathbb{A}_{E} \mathbb{A}_{E 1}^{-1} \hat{\mathbf{1}}\right|^{2}},
\end{aligned}
$$

and obey the sum rule $T_{E}+R_{E}=1$. Using this relation, it is straightforward to verify that the Floquet scattering amplitudes (D14) obey the unitarity conditions (8). For illustration, the transmission function $T_{E}$ is plotted in Fig. 7 for different energies.

\section{Thermodynamic quantities}

Upon inserting the scattering amplitudes (D14) into the general expressions (4), it is now straightforward to determine the mean values of the matter currents $J_{\alpha}^{\rho}$ and the heat currents $J_{\alpha}^{q}=J_{\alpha}^{\varepsilon}-\mu_{\alpha} J_{E}^{\rho}$, which are given by

$$
\begin{aligned}
& J_{1}^{\rho}=-J_{2}^{\rho}=\frac{1}{h} \int_{0}^{\infty} d E\left\{f_{E}^{1}-R_{E} f_{E 1}^{1}-T_{E} f_{E}^{2}\right\} \\
& J_{1}^{q}=\frac{1}{h} \int_{0}^{\infty} d E \zeta_{E}^{q, 1}\left\{f_{E}^{1}-R_{E} f_{E 1}^{1}-T_{E} f_{E}^{2}\right\} \\
& J_{2}^{q}=\frac{1}{h} \int_{0}^{\infty} d E\left\{\zeta_{E}^{q, 2}\left(f_{E}^{2}-f_{E}^{1}\right)+\zeta_{E 1}^{q, 2} R_{E}\left(f_{E 1}^{1}-f_{E}^{2}\right)\right\} .
\end{aligned}
$$

Analogously, the expressions (6a) yields the thermal fluctuations of the matter and heat currents, $D_{\alpha \alpha}^{\rho \rho}$ and $D_{\alpha \alpha}^{q q}=\sum_{u v}\left(\delta_{u \varepsilon}-\mu_{\alpha} \delta_{u \rho}\right)\left(\delta_{v \varepsilon}-\mu_{\alpha} \delta_{v \rho}\right) D_{\alpha \alpha}^{u v}$,

$D_{11}^{\rho \rho}=D_{22}^{\rho \rho}=\frac{1}{h} \int_{0}^{\infty} d E\left\{f_{E}^{\prime 1}+T_{E} f_{E}^{\prime 2}-R_{E} f_{E 1}^{\prime 1}\right\}$

$$
\begin{aligned}
D_{11}^{q q}= & \frac{1}{h} \int_{0}^{\infty} d E \zeta_{E}^{q, 1}\left\{\zeta_{E}^{q, 1}\left(f_{E}^{\prime 1}+T_{E} f_{E}^{\prime 2}-R_{E} f_{E 1}^{\prime 1}\right)\right. \\
& \left.-2 \hbar \omega R_{E} f_{E 1}^{\prime 1}\right\}, \\
D_{22}^{q q}= & \frac{1}{h} \int_{0}^{\infty} d E\left\{(\hbar \omega)^{2} R_{E} f_{E}^{\prime 2}-\left(\zeta_{E 1}^{q, 2}\right)^{2} R_{E} f_{E 1}^{\prime 1}\right. \\
& \left.+\left(\zeta_{E}^{q, 2}\right)^{2}\left(f_{E}^{\prime 1}+T_{E} f_{E}^{\prime 2}\right)\right\} .
\end{aligned}
$$

Finally, the shot-noise contributions to the current fluctuations, $R_{\alpha \alpha}^{\rho \rho}$ and $R_{\alpha \alpha}^{q q}=\sum_{u v}\left(\delta_{u \varepsilon}-\mu_{\alpha} \delta_{u \rho}\right)\left(\delta_{v \varepsilon}-\mu_{\alpha} \delta_{v \rho}\right) R_{\alpha \alpha}^{u v}$, are obtained from Eq. (6b) as

$$
\begin{aligned}
& R_{11}^{\rho \rho}=R_{22}^{\rho \rho}=\frac{1}{h} \int_{0}^{\infty} d E T_{E} R_{E}\left(f_{E 1}^{1}-f_{E}^{2}\right)^{2}, \\
& R_{11}^{q q}=\frac{1}{h} \int_{0}^{\infty} d E\left(\zeta_{E}^{q, 1}\right)^{2} T_{E} R_{E}\left(f_{E 1}^{1}-f_{E}^{2}\right)^{2}, \\
& R_{22}^{q q}=\frac{1}{h} \int_{0}^{\infty} d E\left(\zeta_{E 1}^{q, 2}\right)^{2} T_{E} R_{E}\left(f_{E 1}^{1}-f_{E}^{2}\right)^{2} .
\end{aligned}
$$

In order to evaluate the coefficient (93d) for $x=\rho$ and $\alpha=1$, we further need the mean currents $\tilde{J}_{\alpha}^{x}$ as well as the thermal and shot-noise fluctuations $\tilde{D}_{11}^{\rho \rho}$ and $\tilde{R}_{11}^{\rho \rho}$ for the time-reversed system. These quantities, which are found by replacing $S_{E n, E}^{\alpha \beta}$ with $\mathrm{T}_{\mathbf{B}} \mathrm{T}_{\mathbf{V}} S_{E n, E}^{\alpha \beta}=S_{E, E n}^{\beta \alpha}$ in Eqs. (4) and (6), are given by

$\tilde{J}_{1}^{\rho}=-\tilde{J}_{2}^{\rho}=\frac{1}{h} \int_{0}^{\infty} d E\left\{T_{E} f_{E}^{1}+R_{E} f_{E 1}^{2}-f_{E}^{2}\right\}$,

$\tilde{J}_{1}^{q}=\frac{1}{h} \int_{0}^{\infty} d E\left\{\zeta_{E}^{q, 1}\left(f_{E}^{1}-f_{E}^{2}\right)+\zeta_{E 1}^{q, 1} R_{E}\left(f_{E 1}^{2}-f_{E}^{1}\right)\right\}$,

$\tilde{J}_{2}^{q}=\frac{1}{h} \int_{0}^{\infty} d E \zeta_{E}^{q, 2}\left\{f_{E}^{2}-R_{E} f_{E 1}^{2}-T_{E} f_{E}^{1}\right\}$,

and

$$
\begin{aligned}
& \tilde{D}_{11}^{\rho \rho}=\frac{1}{h} \int_{0}^{\infty} d E\left\{f_{E}^{\prime 2}+T_{E} f_{E}^{\prime 1}-R_{E} f_{E 1}^{\prime 2}\right\}, \\
& \tilde{R}_{11}^{\rho \rho}=\frac{1}{h} \int_{0}^{\infty} d E T_{E} R_{E}\left(f_{E}^{1}-f_{E 1}^{2}\right)^{2} .
\end{aligned}
$$

[1] J. L. Plawsky, Transport Phenomena Fundamentals, 1st ed. (Marcel Dekker, Inc., New York, 2001).

[2] Y. Imry, Introduction to Mesoscopic Physics, 2nd ed. (Oxford University Press, Oxford, 2002). 
[3] R. Landauer, Spatial Variation of Currents and Fields due to Localized Scatterers in Metallic Conduction, IBM J. Res. Dev. 1, 223 (1957).

[4] G. B. Lesovik and I. A. Sadovskyy, Scattering Matrix Approach to the Description of Quantum Electron Transport, Phys. Usp. 54, 1007 (2011).

[5] M. Moskalets and M. Büttiker, Floquet Scattering Theory of Quantum Pumps, Phys. Rev. B 66, 205320 (2002).

[6] M. Moskalets and M. Büttiker, Floquet Scattering Theory for Current and Heat Noise in Large Amplitude Adiabatic Pumps, Phys. Rev. B 70, 245305 (2004).

[7] M. V. Moskalets, Scattering Matrix Approach to Quantum Transport, 1st ed. (Imperial College Press, London, 2012).

[8] K. Brandner, Coherent Transport in Periodically Driven Mesoscopic Conductors: From Scattering Matrices to Quantum Thermodynamics, Z. Naturforsch. 75, 483 (2020).

[9] G. Fève, A. Mahé, J.-M. Berroir, T. Kontos, B. Plaçais, D. C. Glattli, A. Cavanna, B. Etienne, and Y. Jin, An OnDemand Coherent Single-Electron Source, Science 316, 1169 (2007).

[10] E. Bocquillon, F. D. Parmentier, C. Grenier, J. M. Berroir, P. Degiovanni, D. C. Glattli, B. Plaçais, A. Cavanna, Y. Jin, and G. Fève, Electron Quantum Optics: Partitioning Electrons One by One, Phys. Rev. Lett. 108, 196803 (2012).

[11] E. Bocquillon, V. Freulon, J.-M. Berroir, P. Degiovanni, B. Plaçais, A. Cavanna, Y. Jin, and G. Fève, Coherence and Indistinguishability of Single Electrons Emitted by Independent Sources, Science 339, 1054 (2013).

[12] J. Dubois, T. Jullien, F. Portier, P. Roche, A. Cavanna, Y. Jin, W. Wegscheider, P. Roulleau, and D. C. Glattli, Minimal-Excitation States for Electron Quantum Optics Using Levitons, Nature (London) 502, 659 (2013).

[13] T. Jullien, P. Roulleau, B. Roche, A. Cavanna, Y. Jin, and D. C. Glattli, Quantum Tomography of an Electron, Nature (London) 514, 603 (2014).

[14] N. Ubbelohde, F. Hohls, V. Kashcheyevs, T. Wagner, L. Fricke, B. Kästner, K. Pierz, H. W. Schumacher, and R. J. Haug, Partitioning of On-Demand Electron Pairs, Nat. Nanotechnol. 10, 46 (2015).

[15] M. Kataoka, N. Johnson, C. Emary, P. See, J. P. Griffiths, G. A. C. Jones, I. Farrer, D. A. Ritchie, M. Pepper, and T. J. B. M. Janssen, Time-of-Flight Measurements of Single-Electron Wave Packets in Quantum Hall Edge States, Phys. Rev. Lett. 116, 126803 (2016).

[16] C. Bäuerle, D. C. Glattli, T. Meunier, F. Portier, P. Roche, P. Roulleau, S. Takada, and X. Waintal, Coherent Control of Single Electrons: A Review of Current Progress, Rep. Prog. Phys. 81, 056503 (2018).

[17] G. Roussely, E. Arrighi, G. Georgiou, S. Takada, M. Schalk, M. Urdampilleta, A. Ludwig, A. D. Wieck, P. Armagnat, T. Kloss, X. Waintal, T. Meunier, and C. Bäuerle, Unveiling the Bosonic Nature of an Ultrashort Few-Electron Pulse, Nat. Commun. 9, 2811 (2018).

[18] N. Johnson, C. Emary, S. Ryu, H. S. Sim, P. See, J. D. Fletcher, J. P. Griffiths, G. A. C. Jones, I. Farrer, D. A. Ritchie, M. Pepper, T. J. B. M. Janssen, and M. Kataoka, LO-Phonon Emission Rate of Hot Electrons from an
On-Demand Single-Electron Source in a GaAs/AlGaAs Heterostructure, Phys. Rev. Lett. 121, 137703 (2018).

[19] J. D. Fletcher, N. Johnson, E. Locane, P. See, J. P. Griffiths, I. Farrer, D. A. Ritchie, P. W. Brouwer, V. Kashcheyevs, and M. Kataoka, Continuous-Variable Tomography of Solitary Electrons, Nat. Commun. 10, 5298 (2019).

[20] S. P. Giblin, M. Kataoka, J. D. Fletcher, P. See, T. J. B. M. Janssen, J. P. Griffiths, G. A. C. Jones, I. Farrer, and D. A. Ritchie, Towards a Quantum Representation of the Ampere Using Single Electron Pumps, Nat. Commun. 3, 930 (2012).

[21] J. P. Pekola, O.-P. Saira, V. F. Maisi, A. Kemppinen, M. Möttönen, Y. A. Pashkin, and D. V. Averin, SingleElectron Current Sources: Toward a Refined Definition of the Ampere, Rev. Mod. Phys. 85, 1421 (2013).

[22] X. Jehl, B. Voisin, T. Charron, P. Clapera, S. Ray, B. Roche, M. Sanquer, S. Djordjevic, L. Devoille, R. Wacquez, and M. Vinet, Hybrid Metal-Semiconductor Electron Pump for Quantum Metrology, Phys. Rev. X 3, 021012 (2013).

[23] N. Bode, S. V. Kusminskiy, R. Egger, and F. von Oppen, Scattering Theory of Current-Induced Forces in Mesoscopic Systems, Phys. Rev. Lett. 107, 036804 (2011).

[24] R. Bustos-Marún, G. Refael, and F. von Oppen, Adiabatic Quantum Motors, Phys. Rev. Lett. 111, 060802 (2013).

[25] L. Arrachea and F. von Oppen, Reprint of: Nanomagnet Coupled to Quantum Spin Hall Edge: An Adiabatic Quantum Motor, Physica (Amsterdam) 82E, 247 (2016).

[26] A. Bruch, S. V. Kusminskiy, G. Refael, and F. von Oppen, Interacting Adiabatic Quantum Motor, Phys. Rev. B 97, 195411 (2018).

[27] L. Onsager, Reciprocal Relations in Irreversible Processes II, Phys. Rev. 38, 2265 (1931).

[28] L. Onsager, Reciprocal Relations in Irreversible Processes I, Phys. Rev. 37, 405 (1931).

[29] H. B. Callen, Thermodynamics and an Introduction to Thermostatistics, 2nd ed. (John Wiley \& Sons, New York, 1985).

[30] U. Sivan and Y. Imry, Multichannel Landauer Formula for Thermoelectric Transport with Application to Thermopower near the Mobility Edge, Phys. Rev. B 33, 551 (1986).

[31] P. N. Butcher, Thermal and Electrical Transport Formalism for Electronic Microstructures with Many Terminals, J. Phys. Condens. Matter 2, 4869 (1990).

[32] M. F. Ludovico, F. Battista, F. von Oppen, and L. Arrachea, Adiabatic Response and Quantum Thermoelectrics for ac Driven Quantum Systems, Phys. Rev. B 93, 075136 (2016).

[33] H. B. G. Casimir, On Onsager's Principle of Microscopic Reversibility, Rev. Mod. Phys. 17, 343 (1945).

[34] R. Kubo, The Fluctuation-Dissipation Theorem, Rep. Prog. Phys. 29, 255 (1966).

[35] R. Kubo, M. Toda, and N. Hashitsume, Statistical Physics II-Nonequilibrium Statistical Mechanics, 2nd ed. (Springer, Tokyo, 1998).

[36] J. E. Avron, A. Elgart, G. M. Graf, and L. Sadun, Optimal Quantum Pumps, Phys. Rev. Lett. 87, 236601 (2001). 
[37] G. Benenti, K. Saito, and G. Casati, Thermodynamic Bounds on Efficiency for Systems with Broken TimeReversal Symmetry, Phys. Rev. Lett. 106, 230602 (2011).

[38] K. Brandner, K. Saito, and U. Seifert, Strong Bounds on Onsager Coefficients and Efficiency for Three-Terminal Thermoelectric Transport in a Magnetic Field, Phys. Rev. Lett. 110, 070603 (2013).

[39] R. S. Whitney, Thermodynamic and Quantum Bounds on Nonlinear dc Thermoelectric Transport, Phys. Rev. B 87, 115404 (2013).

[40] K. Brandner and U. Seifert, Multi-Terminal Thermoelectric Transport in a Magnetic Field: Bounds on Onsager Coefficients and Efficiency, New J. Phys. 15, 105003 (2013).

[41] F. Mazza, R. Bosisio, G. Benenti, V. Giovannetti, R. Fazio, and F. Taddei, Thermoelectric Efficiency of Three-Terminal Quantum Thermal Machines, New J. Phys. 16, 085001 (2014).

[42] R. S. Whitney, Most Efficient Quantum Thermoelectric at Finite Power Output, Phys. Rev. Lett. 112, 130601 (2014).

[43] B. Sothmann, R. Sánchez, and A. N. Jordan, Quantum Nernst Engines, Europhys. Lett. 107, 47003 (2014).

[44] K. Brandner and U. Seifert, Bound on Thermoelectric Power in a Magnetic Field within Linear Response, Phys. Rev. E 91, 012121 (2015).

[45] R. S. Whitney, Finding the Quantum Thermoelectric with Maximal Efficiency and Minimal Entropy Production at Given Power Output, Phys. Rev. B 91, 115425 (2015).

[46] P. Samuelsson, S. Kheradsoud, and B. Sothmann, Optimal Quantum Interference Thermoelectric Heat Engine with Edge States, Phys. Rev. Lett. 118, 256801 (2017).

[47] K. Brandner, T. Hanazato, and K. Saito, Thermodynamic Bounds on Precision in Ballistic Multi-Terminal Transport, Phys. Rev. Lett. 120, 090601 (2018).

[48] K. Macieszczak, K. Brandner, and J. P. Garrahan, Unified Thermodynamic Uncertainty Relations in Linear Response, Phys. Rev. Lett. 121, 130601 (2018).

[49] R. Luo, G. Benenti, G. Casati, and J. Wang, Thermodynamic Bound on Heat-to-Power Conversion, Phys. Rev. Lett. 121, 080602 (2018).

[50] U. Seifert, From Stochastic Thermodynamics to Thermodynamic Inference, Annu. Rev. Condens. Matter Phys. 10, 171 (2019).

[51] K. Brandner and K. Saito, Thermodynamic Geometry of Microscopic Heat Engines, Phys. Rev. Lett. 124, 040602 (2020).

[52] P. Abiuso and M. Perarnau-Llobet, Optimal Cycles for Low-Dissipation Heat Engines, Phys. Rev. Lett. 124, 110606 (2020).

[53] H. J. D. Miller, M. Scandi, J. Anders, and M. PerarnauLlobet, Work Fluctuations in Slow Processes: Quantum Signatures and Optimal Control, Phys. Rev. Lett. 123, 230603 (2019).

[54] H. J. D. Miller, M. H. Mohammady, M. Perarnau-Llobet, and G. Guarnieri, Thermodynamic Uncertainty Relation in Slowly Driven Quantum Heat Engines, arXiv:2006.07316.

[55] H. J. D. Miller and M. Mehboudi, Geometry of Work Fluctuations versus Efficiency in Microscopic Thermal Machines, Phys. Rev. Lett. 125, 260602 (2020).
[56] M. Büttiker, Scattering Theory of Current and Intensity Noise Correlations in Conductors and Wave Guides, Phys. Rev. B 46, 12485 (1992).

[57] V. Gritsev and A. Polkovnikov, Integrable Floquet Dynamics, SciPost Phys. 2, 021 (2017).

[58] K. Brandner, K. Saito, and U. Seifert, Thermodynamics of Micro- and Nano-Systems Driven by Periodic Temperature Variations, Phys. Rev. X 5, 031019 (2015).

[59] K. Proesmans and C. Van den Broeck, Onsager Coefficients in Periodically Driven Systems, Phys. Rev. Lett. 115, 090601 (2015).

[60] K. Brandner and U. Seifert, Periodic Thermodynamics of Open Quantum Systems, Phys. Rev. E 93, 062134 (2016).

[61] K. Proesmans, B. Cleuren, and C. Van den Broeck, Linear Stochastic Thermodynamics for Periodically Driven Systems, J. Stat. Mech. (2016) 023202.

[62] K. Proesmans, Y. Dreher, M. Gavrilov, J. Bechhoefer, and C. Van den Broeck, Brownian Duet: A Novel Tale of Thermodynamic Efficiency, Phys. Rev. X 6, 041010 (2016).

[63] M. Thomas, T. Karzig, S. V. Kusminskiy, G. Zaránd, and F. von Oppen, Scattering Theory of Adiabatic Reaction Forces due to Out-of-Equilibrium Quantum Environments, Phys. Rev. B 86, 195419 (2012).

[64] C. Texier, Wigner Time Delay and Related Concepts: Application to Transport in Coherent Conductors, Physica (Amsterdam) 82E, 16 (2016).

[65] V. Gasparian, T. Christen, and M. Büttiker, Partial Densities of States, Scattering Matrices, and Green's Functions, Phys. Rev. A 54, 4022 (1996).

[66] Note that $\mathrm{T}_{\mathbf{V}}\left\langle\left\langle\left|\mathcal{S}_{E, \mathbf{V}}^{\alpha \beta}\right|^{2}\right\rangle\right\rangle=\left\langle\left\langle\left|\mathcal{S}_{E, \mathbf{V}}^{\alpha \beta}\right|^{2}\right\rangle\right\rangle$ and $\mathrm{T}_{\mathbf{V}}\left\langle\left\langle\dot{\mathcal{S}}_{E, \mathbf{V}}^{\alpha \beta} \mathcal{S}_{E, \mathbf{V}}^{\alpha \beta}\right\rangle\right\rangle=$ $-\left\langle\left\langle\dot{\mathcal{S}}_{E, \mathbf{V}}^{\alpha \beta} \mathcal{S}_{E, \mathbf{V}}^{\alpha \beta *}\right\rangle\right\rangle$, since $\mathrm{T}_{\mathbf{V}} \mathcal{S}_{E, \mathbf{V} t}^{\alpha \beta}=\mathcal{S}_{E, \mathbf{V}_{-t}}^{\alpha \beta}$.

[67] Recall that $\sum_{n} n\left|S_{E n, E}^{\alpha \beta}\right|^{2} \rightarrow \zeta^{\omega} \operatorname{Im}\left[\left\langle\left\langle\mathcal{S}_{E, \mathbf{V}}^{\alpha \beta} \dot{\mathcal{S}}_{E, \mathbf{V}}^{\alpha \beta *}\right\rangle\right]\right.$ and $\sum_{n} n^{2}\left|S_{E n, E}^{\alpha \beta}\right|^{2} \rightarrow\left(\zeta^{\omega}\right)^{2}\left\langle\left\langle\dot{\mathcal{S}}_{E, \mathbf{V}}^{\alpha \beta} \dot{\mathcal{S}}_{E, \mathbf{V}}^{\alpha \beta *}\right\rangle\right\rangle$ in the limit $\omega \rightarrow 0$.

[68] A. Dechant and S. I. Sasa, Entropic Bounds on Currents in Langevin Systems, Phys. Rev. E 97, 062101 (2018).

[69] M. Büttiker, Coherent and Sequential Tunneling in Series Barriers, IBM J. Res. Dev. 32, 63 (1988).

[70] A. Rossi, T. Tanttu, K. Y. Tan, I. Iisakka, R. Zhao, K. W. Chan, G. C. Tettamanzi, S. Rogge, A. S. Dzurak, and M. Möttönen, An Accurate Single-Electron Pump Based on a Highly Tunable Silicon Quantum Dot, Nano Lett. 14, 3405 (2014).

[71] G. Yamahata, S. P. Giblin, M. Kataoka, T. Karasawa, and A. Fujiwara, Gigahertz Single-Electron Pumping in Silicon with an Accuracy Better than 9.2 parts in $10^{7}$, Appl. Phys. Lett. 109, 013101 (2016).

[72] P. W. Brouwer, Scattering Approach to Parametric Pumping, Phys. Rev. B 58, R10135 (1998).

[73] I. L. Aleiner and A. V. Andreev, Adiabatic Charge Pumping in Almost Open Dots, Phys. Rev. Lett. 81, 1286 (1998).

[74] T. A. Shutenko, I. L. Aleiner, and B. L. Altshuler, Mesoscopic Fluctuations of Adiabatic Charge Pumping in Quantum Dots, Phys. Rev. B 61, 10366 (2000).

[75] J. E. Avron, A. Elgart, G. M. Graf, and L. Sadun, Geometry, Statistics, and Asymptotics of Quantum Pumps, Phys. Rev. B 62, R10618 (2000).

[76] Y. Levinson, O. Entin-Wohlman, and P. Wölfle, Pumping at Resonant Transmission and Transferred Charge Quantization, Physica (Amsterdam) 302A, 335 (2001). 
[77] N. A. Sinitsyn and I. Nemenman, Universal Geometric Theory of Mesoscopic Stochastic Pumps and Reversible Ratchets, Phys. Rev. Lett. 99, 220408 (2007).

[78] E. Potanina, K. Brandner, and C. Flindt, Optimization of Quantized Charge Pumping Using Full Counting Statistics, Phys. Rev. B 99, 035437 (2019).

[79] F. Weinhold, Metric Geometry of Equilibrium Thermodynamics, J. Chem. Phys. 63, 2479 (1975).

[80] P. Salamon and R. S. Berry, Thermodynamic Length and Dissipated Availability, Phys. Rev. Lett. 51, 1127 (1983).

[81] P. Salamon, J. D. Nulton, and R. S. Berry, Length in Statistical Thermodynamics, J. Chem. Phys. 82, 2433 (1985).

[82] B. Andresen, R. S. Berry, R. Gilmore, E. Ihrig, and P. Salamon, Thermodynamic Geometry and the Metrics of Weinhold and Gilmore, Phys. Rev. A 37, 845 (1988).

[83] G. E. Crooks, Measuring Thermodynamic Length, Phys. Rev. Lett. 99, 100602 (2007).

[84] Y. V. Nazarov and Y. M. Blanter, Quantum TransportIntroduction to Nanoscience, 1st ed. (Cambridge University Press, Cambridge, England, 2009).

[85] M. V. Berry, Quantal Phase Factors Accompanying Adiabatic Changes, Proc. R. Soc. A 392, 45 (1984).

[86] Note that the coefficients $g_{\mathbf{V}}^{i j}$ form a symmetric, positive semidefinite matrix and can therefore be consistently identified with a, possibly degenerate, metric in the space of control parameters.

[87] Note that $\gamma_{t}$ is a monotonically increasing and therefore invertible function.

[88] N. Bode, S. V. Kusminskiy, R. Egger, and F. von Oppen, Current-Induced Forces in Mesoscopic Systems: A Scattering-Matrix Approach, Beilstein J. Nanotechnol. 3, 144 (2012).

[89] U. Seifert, Stochastic Thermodynamics: From Principles to the Cost of Precision, Physica (Amsterdam) 504A, 176 (2018).

[90] J. M. Horowitz and T. R. Gingrich, Thermodynamic Uncertainty Relations Constrain Non-Equilibrium Fluctuations, Nat. Phys. 16, 15 (2020).

[91] B. K. Agarwalla and D. Segal, Assessing the Validity of the Thermodynamic Uncertainty Relation in Quantum Systems, Phys. Rev. B 98, 155438 (2018).

[92] J. Liu and D. Segal, Thermodynamic Uncertainty Relation in Quantum Thermoelectric Junctions, Phys. Rev. E 99, 062141 (2019).

[93] A. C. Barato and U. Seifert, Cost and Precision of Brownian Clocks, Phys. Rev. X 6, 041053 (2016).

[94] A. C. Barato, R. Chetrite, A. Faggionato, and D. Gabrielli, Bounds on Current Fluctuations in Periodically Driven Systems, New J. Phys. 20, 103023 (2018).

[95] V. Holubec and A. Ryabov, Cycling Tames Power Fluctuations near Optimum Efficiency, Phys. Rev. Lett. 121, 120601 (2018).
[96] A. Dechant, Multidimensional Thermodynamic Uncertainty Relations, J. Phys. A 52, 035001 (2019).

[97] T. Koyuk, U. Seifert, and P. Pietzonka, A Generalization of the Thermodynamic Uncertainty Relation to Periodically Driven Systems, J. Phys. A 52, 02LT02 (2019).

[98] T. Koyuk and U. Seifert, Operationally Accessible Bounds on Fluctuations and Entropy Production in Periodically Driven Systems, Phys. Rev. Lett. 122, 230601 (2019).

[99] T. Van Vu and Y. Hasegawa, Thermodynamic Uncertainty Relations under Arbitrary Control Protocols, Phys. Rev. Research 2, 013060 (2020).

[100] A. Dechant and S. Sasa, Fluctuation-Response Inequality Out of Equilibrium, Proc. Natl. Acad. Sci. U.S.A. 117, 6430 (2020).

[101] S. Saryal, H. M. Friedman, D. Segal, and B. K. Agarwalla, Thermodynamic Uncertainty Relation in Thermal Transport, Phys. Rev. E 100, 042101 (2019).

[102] Y. Hasegawa and T. Van Vu, Fluctuation Theorem Uncertainty Relation, Phys. Rev. Lett. 123, 110602 (2019).

[103] K. Proesmans and J. M. Horowitz, Hysteretic Thermodynamic Uncertainty Relation for Systems with Broken TimeReversal Symmetry, J. Stat. Mech. (2019) 054005.

[104] P. P. Potts and P. Samuelsson, Thermodynamic Uncertainty Relations Including Measurement and Feedback, Phys. Rev. E 100, 052137 (2019).

[105] G. Falasco, M. Esposito, and J. C. Delvenne, Unifying Thermodynamic Uncertainty Relations, New J. Phys. 22, 053046 (2020).

[106] To derive these expressions, insert the first-order expansions (20) and $S_{E n, E}^{\alpha \beta}=\delta_{n 0} S_{E}^{\alpha \beta}+\Delta \mathcal{Z}_{E n, E}^{\alpha \beta}$ into the Eqs. (A1a) and (A9) and use the sum rule

$$
\sum_{\beta} \operatorname{Re}\left[S_{E}^{\alpha \beta} \mathcal{Z}_{E, E}^{\alpha \beta *}\right]=0
$$

which follows from the unitarity condition (8).

[107] To obtain boundary conditions (D4) and (D5), we require that both $\phi_{E, t}^{\alpha}$ and $p \phi_{E, t}^{\alpha}$ are continuous at the beam splitter, where the second condition ensures the continuity of probability currents [109]. We recall that the momentum operator has the position representation $p \hat{=}-i \hbar \partial_{r_{\alpha}}$ on the leads and $p \hat{=}-i \hbar \partial_{\rho}-\hbar \omega t / l=\hat{P}_{t} / \hbar$ on the loop. The additional factor $(-1)$ on the right-hand sides of the Eqs. (D4c) and (D5c) accounts for the fact that the parametrizations of the leads and the loop run in opposite directions at the connections $\rho=0 \rightarrow r_{1}=0$ and $\rho=0 \rightarrow r_{2}=0$.

[108] F. W. J. Olver, Asymptotics and Special Functions, 1st ed. (Academic Press, New York, 1974).

[109] L. I. Schiff, Quantum Mechanics, 3rd ed. (McGraw-Hill Book Company, New York, 1968). 